\title{
ZUR GESCHICHTE DES GERMANISCHEN CONSONANTISMUS.
}

Kluge hat Beitr. JX, 157-186 im anschluss an Osthoff (Beitr. VIII, 299 f. anm.) gemeingerman. geminaten (verschlusslaute und spiranten) auf eine progressive assimilation von consonanz + nasal $(n)$ zuruckgefuhrt und darin s. 168 'neben laut- und accentverschiebung eines der hauptmomente in der ausbildung der specifisch german. lautform' erkannt. Er hat das gesetz (z. b. s. 174 f.) dahin formuliert, dass nach der wirkung des Vernerschen gesetzes angleichung der betonten $n$-suffixe an die vorausgehenden tönenden spiranten und verschlusslaute eingetreten ist. Der letzte verschiebungsakt hat die einfachen wie die neu entstandenen geminierten medien zu tenues gewandelt, nachdem zuvor die langen (geminierten) spiranten zu langen (geminierten) verschlusslauten geworden waren; man vgl. die tabelle s. 174 anm. 1, vorgerm. *bhugon- (armen. buts s. 168) ergibt in den schwăchsten casus des paradigmas tugn-, das durch das assimilationsgesetz zu bugg- und schliesslich durch die lautrerschiebung zu bukk- gewandelt wurde, vgl. anord. bokkr, ags. bucca, ahd. bock (bocch). Nicht wie bei der westgerm. consonantendehnung hat der folgende stimmbafte laut auf die quantität der vorangehenden consonanz eingewirkt, sondern es ist eine factische totale assimilation eingetreten, der nasal ist in der gemination aufgegangen (vgl. lit. lugnas gebogen : anord. lokkr). Besonders klar tritt dies zu tage in den alten -no- participien, die in historischer zeit als adjectiva erscheinen, wie germ. *fulla- voll für *plná- (vgl. lat. plènus u. a.), alls ganz (neben ala-), ahd. snell, stumm, grimm, stamm (vgl. anord. stammr), andd. hwitt weiss, afries. hwitt (neben hwīt), mndl. wit, witte (neben got. hweils); ahd. flucchi, ahd. mhd. lukki,-e 
(= liukke), nordhumbr. lycce lugnerisch (siehe die anm.) sind parallele bildungen auf suffix -ni (vgl. got. anasiuns), s. Kluge Nominale stammbildungslehre $\$ \S 227-230$. Ebenso sind wahrscheinlich zu beurteilen schweiz. tink feucht, Winteler 8. 62, aus *bingna- (vgl. griech. $\left.\tau \varepsilon^{\prime} \gamma \gamma \varepsilon \iota v\right)>{ }^{*}$ bingga $>{ }^{*}$ binkka; schweiz. teik (mhd. teic), schwäb. toək (flectiert toəke, $k=k k$ ) teig rom obst 1 ) aus vorgerm. dhaizná- (vgl. griech. $\tau o l \chi o \varsigma$ u. a.) > taizza$>$ daigga > daikka; anord. tollr, ags. tol (ll), ahd. zol abgabe aus *tlna-, vgl. Ks. zs. 24, 457, Kluge, Etym. wb. 8. 387, fur afries. tolne, as. tolna (neben $t o l$ ), mnd. toln ist möglicherweise doch fremder einfluss zuzugeben; abd. scarf, sarpf, scarpf scharf, as. scarp, ags. scearp, anord. skarpr beruhen auf *skrtoná- >

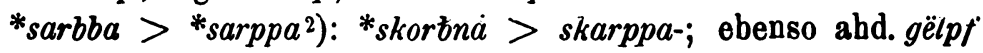
vgl. Graff IV, 196 f., mhd. gelf, gelpf tubermutig gegen anord. gjálfr sonitus u. a.; ahd. erpf, anord. jarpr fuscus; vielleicht auch mhd. kluoc, anord. klókr $(k=k k)$; anord. goltr geschnittenes schwein, ahd. galza, mhd. galze (aus *ghaltná-), nhd. gälze gegen anord. gelda verschneiden, ahd. galt, neubd. dialektisch gelt nicht trächtig; nhd. leck neben älter nhd. lech, dieses dial. 7. b. kărntisch D. M. III, 309 aus *lezná- (vgl. lit. laszas tropfen) $=$ anord. lekr ${ }^{3}$ u. a. Das hauptgebiet dieser assimilationserscheinung ist die schwache nominalflexion und ausserdem die verbale intensivbildung, vgl. Kluge, Beitr. IX, $170 \mathrm{f}$. Diese letztere schliesse ich, als in andern zusammenhängen stehend, aus, glaube aher die untersuchung fur die nomina noch einmal auf-

1) Aus frünk. mundarten kenne ich auch formen mit - z-, dem alten einfachen laut entsprechend, ebenso mhd. lüge, andd. luggi lugnerisch gegen northumb. lycce.

9) Dass die grundform mit $r$ son. anzusetzen ist, beweist die ahd. nebenform sarp $f$, in der $c$ ganz wie in ahd. solan (neben scolan) ans scl- geschwanden ist, in der ablautsform mit stufe -o- (also "scorbina- = scarpf) musste $c$ bewahrt bleiben, zu sarpf ist das ganz analoge prät. ags. salde Matth. 27, 31; Sievers, Beitr. IX, 562 zu vergleichen, wenn es auch wabrscheinlich ist, dass der vocal auf ausgleichung beruht und ahd. solda im Trierer capit. ursprünglicher ist. v. Fierlinger, Ks. zs. 27, 191 hat mit unrecht ahd. sarpf von scarpf getrennt; ags. as. anord. $p$, ahd. $f$ in diesem worte stellen die vor consonanz gekulute stammform dar, vgl. a. s. 512 ff.

$\left.{ }^{3}\right)$ Wegen $k=k k$ vgl. unten s. 513, von lekr abgeleitet ist leka lech sein. 
nehmen zu mlussen, da sich mir einzelnheiten ergeben haben, die mit dem Klugeschen gesetze gar nicht oder nur mittels kunstlichster associationsbildungen in einklang zu bringen sind.

Mein ausgangspunkt sind einzelne formen heutiger mundarten gewesen, so namentlich schweizerische, wie sie teils bei Winteler teils in der kurz erschienenen dissertation von A. Bachmann, Beiträge zur geschichte der schweizerischen gutturallaute, Zurich 1886 verzeichent sind, einzelnes ausschlaggebende kommt auch aus dem schwăbischen und andern mundarten hinzu. Indessen ist bezluglich der letzteren grösste vorsicht geboten. Der consonantenstand des schweizerischen ist durch die beobachtungen Wintelers unzweideutig festgestellt, für das schwäbische, als meinem heimatsdialekt, kann ich gleichfalls gesicherte angaben machen, ich habe mich um weitere belege schon deswegen nicht sehr bemuht, weil sie in einer orthographie gegeben zu werden pflegen, die sie zu allen schlussen, wenn es not tut, verwerten lässt. Heinzerlings Siegerländer mundart macht eine ruhmliche ausnahme, manche andere sehr verdienstliche darstellung mit einer an sich durchsichtigen transscription hat darauf verzichtet sich mit diesen complicierteren fragen des consonantismus, in denen bei oberflăchlicher betrachtung meist ausnahmen von der lautverschiebung vorzuliegen schienen, abzugeben, oder mehr als die stehenden paradebeispiele aufzufuhren.

Was die methode der untersuchung anlangt, so war ich bestrebt, so weit als nur irgend möglich die erfahrungsmasssigen beutigen dialektischen bildungen zu grund zu legen. Ich bin durchaus nicht der meinung, dass dieselben z. b. mit den ahd. sich decken (es ist vielmehr sebr davor zu warnen, sich von den heutigen lautrorstellungen beherrschen $\mathrm{zu}$ lassen), die gerăuschlaute mögen nach intensităt, einsatz, quantităt u. \&. w. andere geworden sein, allein wir haben keine spur davon, dass sie aus dem alten geleise herausgetreten wären, und was das wichtigste, so viel ich sehe, ist die innere harmonie der einzelnen gruppen von leicht constatierbaren einzelnheiten abgesehen (die naturlich nicht ohne einfluss auf die totalităt der sprachlaute gewesen sind) wenig alteriert worden; doch kann dafur den beweis widerum nur die folgende untersuchung selbst liefern. So wissen wir z. b. von den 
heutigen rheinfränk. u. a. mundarten, dass das lautgesetz einer allgemeinen intensitätsreduction den consonantismus verändert hat, allein dadurch ist das alte verhăltnis z. b. des einfachen zum geminierten laut nicht verwischt sondern genau dasselbe geblieben, weil beide in gleicher weise von dem gleichen geschick betroffen sind. Darin besteht uberhaupt beinahe die gesammte methodologie der mundartlichen historischen forschung, bei beurteilung einer jeden einzelnen form sich möglichst in zusammenhang mit dem specialtypus der einzelmundart zu Lalten, und derselbe ist meist nicht schwer festzustellen.

1. Die von Kluge gesammelten belege zerfallen in zwei categorien: a) Die form mit gedehnter') consonanz hat sich allein behauptet, sei es dass tuberhaupt kein wechsel in der consonanz möglich war, da $-n$ bei endbetonung in allen fällen unmittelbar auf den verschlusslaut oder spiranten des stammes folgte, oder dass der assimilierte doppellant den einfachen analogisch verdrăngte. Im letzteren fall waren widerum zwei möglichkeiten: entweder wurde aus den casus in denen assimilation des ableitenden - $n$ eintrat, tuberhaupt ein paradigma $n$-loser flexion gebildet, vgl. Osthoff, Beitr. VIII, 300 anm., z. b. nach gen. pl. *bukko (aus *bugnō') wie *dazj ein nom. sg. *bukk-z (statt *buko 8. 0.) wio *daz-z; oder es wurden nach massgabe der casus mit $n$-flexion auch die geminierten formen in diesem system belassen, z. b. nom. sq. "bukko statt *buko (danach ein neuer gen. pl. *bukk-nō u. a.).

In der entscheidung zwischen diesen mōglichkeiten stimmen vielfach die einzelnen altgerman. dialekte nicht uberein, vielmehr ist im einen idiom die starke, im andorn die schwache flexion zum durchbruch gekommen, es lassen sich nur ganz wenige beispiele ausfindig machen, in denen gleichmassigkeit berrschte, vgl. anord. bokkr, ahd. bocch : ags. bucca, anord. bukki gegen anord. stokkr, ags. stocc, ahd. stocch stock.

b) Die form mit doppelconsonanz ist uberhaupt nur auf westgerman. boden zu belegen und erscheint teils

1) Ich wage nicht, liber die phonetische geltung der gemeingerm. assimilierten verschlusslaute mich definitiv zu äussern, wenn es mir auch wahrscheinlich ist, dass wirklich lange consonanz, nicht geminata entstanden ist, womit sich anscheinend am leichtesten verträgt, dass nnter bestimmten bedingangen einfache (gekürzte) consonanz erscheint. 
innerbalb desselben gebiets, teils durch beiziehung got. und scand. belege gleichzeitig mit einfachem consonanten, vgl. ahd. rokko : ags. ryge, anord. rugr; ags. sceaza, sceacza (?) : anord. skagi (ahd. scahho mit grammat. wechsel) u. a. In diesen und ihnen analogen fällen ist therhaupt kein ubertritt in die starke flexion zu constatieren, sondern die stammform mit gemination ist ausnahmslos in der schwachen declinationsclasse verblieben. Es ist demnach kein äusseres anzeichen vorhanden, dass $-n$ in der gemination untergegangen wäre, im gegenteil für alle diese falle ist durch die stetigkeit des flexionscharakters die fortexistenz desselben gesichert. Die beschränkung dieser categorie auf die westgerman. sprachstämme, wie der in denselben worten aus derselben periode nachweisbare wechsel einfacher und doppelter consonanz, macht es an sich wahrscheinlich, dass diese erscheinung auf denselben oder analogen bedingungen berubt wie sie in der sogenannten west. germ. consonantendehnung begrundet liegen; ein wechsel wie ahd. troffo : tropfo gegen ags. dropa, anord. dropi ist an sich genau identisch mit dem in sleifen : sleipfen Beitr. VII, $123 \mathrm{f}$; oder nhd. sprosse : sprotze wie mhd. weize : veize u. a.

2. Nur für die unter 1.a) charakterisierten fälle ist Kluges gesetz von der verschiebung der geminierten medien resp. tön. spiranten zu geminierten harten verschlusslauten in gemeingerman. periode glatt durchzufuhren. Gerade unter den sub 1. b) besprochenen nur westgerm. geminaten erscheint eine betrăchtliche zahl westgerm. geminierter medien ${ }^{1}$ ) oder spiranten die aus der Klugeschen verschiebung heraustreten. Kluge hat allerdings eine theorie aufgestellt, wonach sie doch damit vereinbar sein sollen, es ist folgende. Um die nur hochdeutsche gemination in ahd. chnappo, mhd. knappe (doch vgl. auch afries. knappa neben knapa) neben ahd. chnabo, mhd. knabe und ags. cnapa, seltener cnafa, as. knapo, anord. knapi zu deuten, sieht or sich a. a. o. s. 176 zur erklärung veranlasst: 'Dass neben ahd. chnabo eine form *knapp- (d. b. gemeingermanisch) aus *knabn- denkbar ist, ergibt sich aus dem bisherigen. Diese doppelformen fuhrten durch association zu zwei neuen

1) Ueber anord. geminierte medien vgl. u. s. 520. 
formenpaaren: man bildete zu *knato eine neue geminationsform *knabba oder zu der geminierten form *knapp- im anschluss an *knabo eine form mit einfacher consonanz *knapa: jenes ist das mbd. knappe, dies das ags. cnapa.' Eine derartige formschöpfung halte ich für nicht vereinbar mit den allgemeinen anschauungen, die sich für die associationsbildungen der lebenden sprache festgesetzt haben. Gewöhnlich vergleicht man dieselben mit der arithmetischen auffindung der vierten proportionale, bei drei gegebenen grösseu; so trifft fur die entstehung des gemeingerm. nom. *bukkz (gegen *bugon-) die ansetzung der gleichung zu: gen. pl. ${ }^{*} d a z \bar{o}:$ gen. pl. ${ }^{*} b u k k \bar{o}=$ nom. sing. *daz-z : nom. sg. *bukk-z. Im vorliegenden fall dagegen weiss ich kein tertium aufufinden, das die schlussfolgerung ermöglichte. Fur das sprachgefuhl, wenn ich die formale kraft so bezeichnen darf, liegen die beiden data vor: *knat (mit stim mhaftem reibelaut) und *knapp (mit langem, stimmlosem, hartem verschlusslaut), tertium non datur. Eine proportion wäre möglich, wenn entweder ein *knabb oder ein *knap existierte: knabb : knat = knapp : knap oder knap $: k n a p p=k n a t: k n a b b$ (um die vierten proportionalen gleich einzusetzen). Nachdem *knat und *knapp im stammausgang so sehr weit auseinandergegangen waren, kōnnte ein *knabb psychologisch nur so entstanden gedacht werden, dass mit bewusstsein reflectiert worden wäre, worin die differenz $t: p p$ bestunde, und es erscheint mir sehr fraglich, ob das resultat dieser reflexion dasselbe wie bei Kluge gewesen wäre, denn $\zeta$ und $p p$ (als gesprochene laute) verhalten sich nicht wie einfache und doppelconsonanz. Ich glaube danach, dass der theorie Kluges in keinerlei weise raum gestattet werden darf. 1)

1) Noch bedenklicher wird dieselbe bei langsilbigen stämmen, wenn z. b. zu einer doublette hēzo: hékko haken ein *héggo angesetzt wird, denn nach dem gesetz von der kürzung der consonanz nach langer silbe musste hekko noch gemeingerm. zu *héko werden, was jedenfalls in nicht zu grossem zeitlichem abstand von der entstehung der gemination zu denken ist, und bei einer doppelheit hezo : hêko bleibt für *hëggo keine möglichkeit. Vgl. a. a. o. s. 184, wo Kluge in solchen bildungen 'jiingere reflexe der sonst weit verbreiteten consonantendehnung nach kurzen vocalen' sieht, was nur auf dem papier denkbar ist. 
3. Einzelne wortformen:

a) Schwăb. baokə eine art trommel bei kirchenmusik, (jetzt existiert das instrument meines wissens nicht mehr, auch das wort scheint nur noch wenig gekannt zu sein) von mhd. buke pauke, schwäb. pauk, die wahrscheinlich wurzelverwant sind, zu trennen, ist offenbar mit ags. beacen signum, afries. bēken (aus *baukin) feuersignal, ahd. bouhhan, pouchan etc., vgl. Graff III, 44, identisch; weiteres bei Möller, Ks. zs. 24, 439 ff. Schwäb. $k$ (hauchlose fortis) ist notwendig westgerm. $k k$ oder gg. Urgerman. gemination ist in diesem worte nach Kluge s. 169 nicht denkbar, weil wurzelbetonung angenommen werden muss, jedenfalls setzt aber schwăb. baoks eine ahd. doublette bouhhan (= beácen) : boucchan, boukkan voraus.

b) Ganz analog wie der auf der westgerm. consonantendehnuug beruhende wechsel zwischen schweiz. akchar : achar besteht im schweiz. ein solcher zwischen brokcha : brocha brocken, balkcho : balchə balken, wulkchə : wulche wolke, Bachmann a. a. o. s. 40. Letzteres ist nur westgerm., das $-n$ der ableitung ist nach ausweis von ags. wolcen, as. wolkan nicht zu grunde gegangen, $k$ in der verbindung $-l k$ ist im hochalem., woher die belege stammen, zu ch geworden, -kch setzt notwendig -lkk voraus, das aber nicht aus urgerm. periode stammen kann; weiteres uber das wort s. $u$.

c) Mlat. hupa hopfen, sei es nun aus dem german. entlehnt oder selbst stammwort (was mir das wahrscheinlichere ist), weist auf ein *hupo zurlick, das fur ahd. hopfo, ndl. hoppe, mengl. hoppe als grundlage der gemination anzusetzen ist und das doch wol wegen der abweichenden benennung der pflanze im anord. (zu anord. humal ist vielmebr mlat. humlo zu vergleichen) doch nicht in urgerm. zeit existiert haben wird, dio gemination in den westgerm. formen muss also jungern datums als die gemeingerm. assimilation sein. Ganz sicher jungeres lehnwort ist lat. draco, griech. $\delta \rho \alpha ́ x \omega \nu$ drache, wie ags. draca ausweist. Im ahd. bestehen die doppelformen: trahho : traccho, mhd. drache : dracke, letzteres auch in den heutigen mundarten, vgl. Schmeller, Wb.2 I, 565, Munda. s. 87; DM. III, 16; Weinhold, Bair. gr. s. 189, hier ist jeder gedanke an urgerm. assimilation ausgeschlossen.

Weiteres werden die folgenden zusammenstellungen ergeben. 
Meine meinung, die ich zu begrunden suchen werde, geht dahin, dass in westgerm. sprachperiode im zusammenhang mit der dehnung die durch folgendes $i, n, r, l$ bewirkt wurde, auch - $n$, (m) derableitung den vorangehenden consonanten gedebnt hat.') Die urgermanische assimilation, deren bildungsgesetz Kluge festgestellt hat, verliert dadurch eine reibe von belegen, die derselbe im beweismaterial vorgefuhrt hat, so dass ich mich veranlasst sehe, die mir bekannten fälle hier zusammenzustellen, eine anzahl -no-participien sind bereits oben s. $504 \mathrm{f}$. aufgefubrt.

\section{Die german. consonantendehnung.}

a) Die vorbedingung für die angleichung des nasals an den verschlusslaut oder spiranten des stammes ist suffixbetonung. Es lassen sich einige falle nachweisen in denen diese stattgehabt hat, ohne dass die assimilation eingetreten wäre; sie sind von Kluge in dem artikel 'Labialisierung der idg. velaren tenuis im germ.' Beitr. XI, 560 ff. behandelt. Isl. ags. hrogn, ahd. rogan, neben rogo (vgl. daruber Kluge, Beitr. IX, 169 f.), schwed. rom, gutn. rumn (letztere =*hrofn); got. auhns, anord. ofn - ogn, westgerm. *ofn aus *uqnó- ofen, vgl. griech. isvós (uber die entsprechung vgl. Noreen, Beitr. VIl, 433 f.), wol auch ahd. zwĩfo (aus grundform *dweign-): ahd. zwëho (grdf. *dwiqon-). Die Klugesche regel, wonach der labialismus eintritt, 'wenn innerhalb des wortkörpers ein $w$ oder ein anderer labial erscheint2)', reicht nur fur die minderheit

1) Kluge 8. a. o. 8. 149 nimmt stillschweigend $n$ unter die westgerm. geminierenden laute auf, ohne es in der folgenden abhandlang zur sprache zu briñgêên óder zu verwerten. Auch von andern forschern ist mehrfach schon beiläufig $n$ - als geminationswirkend angesetzt worden, vgl. z. b. Kugel, Ueb. d. keron. gl. s. 78, Franck, Mnl. gr. \$\$ 94. 95. - Bachmann, der in seiner oben s. 506 verzeichneten dissertation sehr wertvolles material geliefert hat, ist bei der historischen erklärung desselben im hőchsten grade unmethodisch verfahren; man vgl. s. 31: 'Sehen wir die umgebung väher an, in welcher das $k$ auftritt, so machen wir die entdeckung, dass mit wenigen ausnahmen das $k$ stets (doch vgl. s. 19 $\alpha$ ) nach oder vor liquida, nasal oder (nach) langem vocal erscheint. Diesem umstant werden wir auch die gemination zuzuschreiben haben.'

2) Danach hätte got. auhns : anord. ofn, westgerm. *ofn von Kluge 
der fălle aus, doch bedarf die frage einer systematischen untersuchung, die sich auf umfassenderes, in den heutigen mundarten reichlich vorhandenes material zu stützen hătte.1) Bis dahin wären die obigen 'ausnahmen' in suspenso zu lassen.

b) Zur klarstellung einer reihe von entsprechungen ist noch auf folgendes aufmerksam zu machen. Kluge hat Beitr. IX, $178 \mathrm{ff}$. im anschluss an Osthoff nachgewiesen, dass die germanische geminate nach langer silbe verein facht worden ist, ich glaube dass dieselbe reduction auch vor consonanz (bei vorangehender kürze) erfolgen musste. Fưr all die fälle, in denen $n$-flexion aufgegeben wurde, ist im nom. sg. nach wirkung des synkopierungsgesetzes die geminate mit dem nominativ $-z(r)$ zusammengestossen (resp. in den auslaut getreten), dabei wurde sie zum einfachen laut gekturzt und ebenso in sämtlichen flexionsformen, in denen consonant mit consonant zusammenstiess. Dem scheint das anord., vgl. z. b. lokkr gegen ahd. loc (pl. locchar), ags. loc (gen. locces) locke, direct zu widersprechen, wåhrend formen wie ags. hraep, gen. hnaeppes napf, afries. kop, koppes kopf, sket, dat. skette schatz, ahd. scof : scopf, ags. sceop u. a. zweifellos dafur zeugen, wenn auch auf westgerm. boden die reduction im absoluten oder silben-auslaut eingetreten sein wird, vgl. Sievers, Ags. gr. ${ }^{2}$ \& 231, 1. 2. 3. Was die schreibung im altnordischen betrifft, 80 ist es aus

unter die zutreffenden belege aufgenommen werden kőnnen, vgl. Noreens FÉx Fvos a. 8. 0 .

1) Selbst in germ. fimf, idg. *penqe scheint die labialisierung an bestimmte bedingungen geknlipft zu sein, wie mundartliche formen mit inl. velar beweisen: schwäb. fuchze 15, fuchzk 50, vgl. Birlinger, Alem. sprache rechts des Rheins 8. 178, Augsb. wUrterb. 8. 149; Weinhold, Bair. gr. \& 184; fauchzk kenne ich aus dem grossherzogtum Sachsen. In schwäb. hat das ordinale die auffällige form fuft der flinfte, ebenso fufzz, fuf $z k 15,50$, wo ein ursprïnglicher nasal unmöglich geschwunden sein kann. Nimmt man an (vgl. K8. 2s. 27, 193 f.), dass eine alte doppelheit idg. *penqe fünf : "pnqto- der fünfte bestanden hat, so entspräche ein germ. fimf: fühta (aus *funhta), in schwäb. fuft wäre $f$ aus fimf eingeschleppt, während in fuchze, fuchzk, sïchs. fauchzk der guttural bewahrt blieb. - ch aus sechzehn, sechzig stammen zu lassen, was nahe läge, verbietet der schwund des nasals; die kürzung im schwäb. von $\bar{u}: \breve{u}$ ist ohne anstand. Es ist nicht unmöglich, dass auch mnl. vichtiene fünfzehn, vichte der fünfte neben vijftich fünfzig, vijfte der fünfte auf den alten wechsel zurtickweisen, vgl. Franck, Mnl. gram. \$109. 
allgemein phonetischen gründen sehr unwabrscheinlich, dass z. b. in $-k k r,-p p r$ etc. eine dreifache d. h. drei moren messende consonanz olne silbengrenze sollte gesprochen worden sein. Sierers hat ja bekanntlich nachgerviesen, dass in den skandinavischen rhythmen das nom. $-r$ nicht silbenbildend, sondern stimmlos war. Mit diesen zweifeln begegnet sich aber auch die orthographie der denkmäler. Aus Hofforys oldnordiske consonantstudier 8. $38 \mathrm{ff}$. constatiere ich ${ }^{1)}$ : 1. nach langem vocal: hlátr (ahd. hlahtar), slátr (stamm *slahtra-), látr (stamm *lahtra-), dótr dat. sg., nom. acc. pl. u. a. (vgl. s. 45) zu dóttir (urn. dohtriR), nétr nom. acc. pl. zu nótt nacht (die unterscheidung, ob nord. synkope, oder ursprtingliche folge, cons. + cons., vorliegt, ist für uns ganz gleichgultig, vgl. ubrigens Brate, Beitr. $\mathrm{X}, 75 \mathrm{f}$ ). 2. Nach kurzem vocal: vetr (got. wintrus) gegen mottul mantel u. a.; -vetna (zu vetta) in hvarvetna, hvatvetna 8. 46. Auf grund dieses bestandes schliesst Hoffory s. 41, dass $t t$ vor consonant in $t$ ubergegangen sei, und kntipft die vermutung an, dass dasselbe fur die urgern. $t t$ wie uberhaupt fur såmtliche $k k, p p, d d, s s$ zu gelten habe, indem sie unter denselben bedingungen zu $k, p, d, s$ geworden sein mulssten. So hat demnach bereits Hoffory s. $43 \mathrm{ff}$. die hier erforderlichen formen nom. sy. skatr (dat. skatti), lokr (dat. lokki), topr (dat. toppi), kotr (dat. ketti), bekr (pl. bekkir), kokr (dat. kekki), drótr (dat. dretti) u. a. vorausgesetzt, mit denen auf derselben stufe stehen: dat. sg. drótni, pl. drótnar etc. (nom. sg. dróttin) s. 52. Vgl. noch aus der adjectivflexion s. 53 ff.: rétr (acc. réttan), rétri, rétrar, rétra u. a.; ebenso skakr, skakri, skakrar, skakra u. a. zu skakkir, krapr, krapri, kraprar, krapra u. a. zu krappir u. 8. w. Dio orthographio des Stockholmer homilienbuchs verhält sich nun dazu folgendermassen vgl. s. 69 ff.: dróttin $322 \mathrm{mal}$, drótinn, drótin, drótins $15 \mathrm{mal}$, drótni 34, drótnar 5, drótna (gen. pl.) 1 mal, dagegen dróttni 6, dróttnar $1 \mathrm{mal}$. Ferner hlátr $6 \mathrm{mal}$, nie *hláttr, dótr $6 \mathrm{mal}$, dóttir, dóttur $7 \mathrm{mal}$, ncétr 4, nótt sehr häufig; vetr durchweg

1) Es muss hervorgehoben werden, dass es sich dabei nur um geminaten speciell skandinavischer provenienz handelt, mit den unten folgenden belegen für urgermanischen doppellaut znsammen ergibt sich aber $\mathrm{zweifellos,} \mathrm{dass} \mathrm{keine} \mathrm{articulationsverschiedenheit} \mathrm{zwischen}$ beiden bestanden hat, vgl. zur ganzen frage die besprechang von Mogk, Anz. fua. X, 61 ff. 
bis auf das einzige vettre $215^{1}$ u. s. w. Ich fubre noch an: stok (acc. sg.) 10125, aber stockar 10124, ocr 1. mal, ycr 2 mal, ycrar $1 \mathrm{mal}$, ebenso $1 \mathrm{mal}$ yckar, daneben 'unorganisch' yckrum, okor je einmal; drucner 4, drucnom 1 gegen je 1 drucken, drucket (drucnar in den von Sievers ed. Frostuthingslög fragm. 8. 15) u. s. w. Noreen bringt noch bei apr (aus *appr), schwed. amper hart, Anorw. und aisl. gr. § 208.

Dadurch ist das gesetz von der kürzung langer zu einfacher consonanz vor folgendem consonanten auch fur das skandinavische erwiesen und es muss zunächst auf fälle wie anord. freknur, norw. fraknor, schwed. fräkner, dăn. fregner, engl. frecken, fries. (Amrum) friakan sommersprossen, norweg. fraknätt sommersprossig u. a. (vgl. Möller, Ks. zs. 24, 460 ff.) angewendet werden; diese wörter sind von Bugge, Ks. zs. 19, 438 bereits mit aind. prçiš bunt, lit. prëszas mal, flecken,

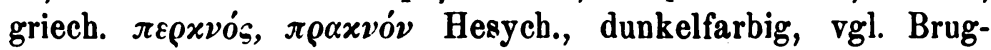
mann, Grundriss $\$ \S 292$. 306, identificiert worden, $-x \nu$ - des griech. reflectiert im germ. als $-k k$ - (aus $-z^{n-}$ ) das vor dem neuantretenden $-n$ der schwachen flexion reduciert wurde ( $\nabla g l$. oben drucnar : druckin). Ferner anord. spakr kann nicht von as. spāhi, mhd. spaehe einerseits, noch von aind. spac-, lat. spec(in speculum etc.) andererseits getrennt werden, geht demnach auf ein urgerm. *spazna- $>{ }^{*}$ spakka- $>$ anord. spakr zurttck; gerade in der adjectivflexion musste in 10 casusformen (vgl. Hoffory a. a. .0. s. 53 f.) die reduction erfolgen und es war auf dieser grundlage leicht eine verallgemeinerung der einfacben articulation möglich, anders Brugmann, Grundr. $§ 469,7$. Dieselbe erklärung nehme ich fur anord. frekr, got. friks (in faihufriks), vgl. gemin. in abd. frecchi avaritia Murb. hymn. Sievers 8. 16, ahd. freh, ags. freca neben frecca als ableitung, in anspruch, Joh. Schmidt, Idg. vocal. I, 64 hat gewiss recht die idg. wurzel prek in dem worte zu vermuten und got. fraihnan etc. damit zusammenzustellen, vgl. anord. lekr oben s. 505 . Weiteres wird sich unten ergeben.

Während diese kurzung sicher erst in der separatentwicklung des skandinavischen eingetreten ist, halte ich es für sebr wahrscheinlich, dass fur die westgerm. zweige dieselbe in der gemeinsamen sprachperiode erfolgte. Der wechsel des geminierten mit dem einfachen cononanten spiegelt sich gut im ahd. 
wider: ahd. scof : scopf, ags. sceop dichter; ahd. chrof : chropf kropf, ahd. chnof : chnopf knopf, ahd. sarf, scarf, ags. scearp, anord. skarpr : ahd. sarpf, scarpf, ahd. stoch : stocch stock u. a. Ueber die ags. reduction handelt Sievers, Ags. gr. ${ }^{2} \S 231$. Kluge statuiert für diese fälle einen wechsel in der stamnbildung (vgl. s. 171) zwischen $n$ - und $n$-losem suffixe, was mir nicht glaublich erscheint, germ. *alla : *ala sind in sofern nicht gleich zu beurteilen, als für die lautliche doppelheit von alters her eine functionsdifferenz bestand, wodurch die verschiedenheit der stammbildung begreiflich wird.

Die belege fur assimilation sind nun:

A. anord. lokkr, ags. loc (locces), afries. pl. lokkar, ahd. loc (loccha) locke, vgl. lit. lugnas biegsam, gebogen, 8. 1691); anord. bokkr, bukkr, bokki, ahd. mhd. nhd. bock, ags. bucca, 8. 165. 169; - ags. frocca, anord. fraukr frosch (uber ags. frocza u. 8. 521) s. 167. 184; - ahd. floccho, mhd. vlocke, ndl. vlok, dăn. flokke, schwed. flokka, engl. flock, mit anderer ablantsstufe anord. floki flocke, s. 165. 173. Dazu ahd. facchi flugge Beitr. VIl, 134 anm., anord. flokkr, ags. flocc herde, schwarm; - ags. raecc, anord. rakki spurhund; - ahd. roccho, anord. rokkr, mittelengl. rocke (ags. * rocca) rocken s. 165. 169; - ahd. smoccho, ags. smocc, anord. smokkr unterkleid 8. 165. 169. 173, Beitr. VII, 133 anm. 2; - ahd. flëccho, flëk, mhd. vlëcke, vlëc, anord. flekkr fleck s. 165, dazu anord. flik (wie oben floki) und abgeleitet anord. flikki, age. flicce speckseite; - ahd. nacch, mhd. nac (gen. nackes), anord. hnakki, ags. hnecca (mit ablaut, vgl. engl. neck), afries. hneckn nacken s. 165; - ags. hreák haufen (aus *hraukk-), anord. hraukr die einfacho consonanz in anord. hruga, vgl. air. cruach Beitr. X, 444; - ahd. smac(ch), ags. smaec (cc), afries. smek (smekkes gen.) geschmack s. 167, Beitr. VI, 93 anm. 4. VII, 134; - ahd. stoc (ch), mhd. stoc, stoch, anord. stokkr, ags. stocc, afries. stok (dat. stokke) stock 8. 167 ; - ndd. fäken oft Kluge s. 182 zu griech. $\pi v x v \alpha \dot{~}$ oft (uber md. fache oft, facher öfter s. u. s. 524); - ags. $\operatorname{coc}(c)$, engl. cock, anord. kokkr hahn, Osthoff, Beitr. VIII, 297; schweiz. blokch, abd. bloch, mbd. bloch, schweiz. bloch block

1) Die seitenzahlen verweisen auf den artikel Kluges und geben den ort, wo das betr. nomen von Kluge behandelt ist. 
Bachmann s. 55; DM. IV, 206; - schweiz. rikch heftel von faden, mhd. ric (-ckes) Beitr. VII, 133 anm. 2 gegen ahd. riga, mhd. rige, rîhe, weiteres siehe Bacbmann s. 50; - ebenda schweiz. bukch hligel, schwab. buk aufbiegung; - schweiz. bikcha, bëkcha spitzige hacke zu ahd. bīhal beil Bachmann s. $52^{1}$ ); - anord. bekkr, ags. becc, as. anfr. beki, ahd. bah, mhd. bach $^{2}$ ) bach s. 167 ; - ahd. rucch- neben rouh rauch 8. 167, ebenda ahd. bizucch-; - schweiz. flock felsabhang, gegen ags. flōh, ahd. fluah rupes; - got. pairko loch (zu pairh) vgl. s. 182; - ags. wicca zauberer (gegen wīzlère) 8. 165; - ahd. drëch, anord. brekkr, afries. threkk dreck ${ }^{3}$ ); - hier schliessen sich die von Paul, Beitr. VII, $133 \mathrm{f}$. anm. behandelten nomina an; vgl. ferner ags. afries. as. $w \bar{\imath} c$ Kluge s. 183; ags. hōcor gegen ahd. huoh, ags. hōh spott s. 184 .

') Es ist danach nicht nötig einen idg. wechsel im stammausgang der wurzel anzunehmen.

2) Auf hd. gebiet z. b. im bair. bezeugen noch eigennamen mit dem compositionsglied -beck die gemination. S. 171 sieht Kluge die verschiedenheit des consonantismus in der stammbildung begründet und nimmt fiir *bakki (neben *baki) suffix -ni an. Ich habe mich bereits oben s. 515 dagegen ausgesprochen, schwierigkeit kann nur as. beki bereiten, abd. bah ist die lautgesetzliche nominativform, deren consonanz verallgemeinert worden ist: ${ }^{*} b a k k>{ }^{*} b a k>b a h$, plur. ${ }^{*} b e k k i$. Vgl. die altels. -baci, bac (c kann nur kk vertreten) bei Socin, Strassburger studien I, 234. 251. Auch auf ndd. boden sind ortsnamen auf -becke (ohne dehnung des wurzelvocals) reichlich vorhanden; das simplex bekke belegt z. b. Jellinghaus, Zur einteilung der ndd. mundarten 8. 37. As. beki (das tibrigens nur aus ortsnamen, meines wissens, belegt ist) kann darum kaum anders als wie hd. bah aus einem westgerm. *bak-z (ans *bakkiz) erklärt werden, diese selbe form muss anch für ags. becc notwendig vorausliegen, der umlaut stammt aus den obl. cass. So bestand die differenz nom. sg. *bak, pl. *bekki, die wie im hd. zu *bak: *beki umgebildet wurde (acc. pl. beki in den anfr. psalmen als einziger beleg). Da nun aber im formsystem der $i$-decl. keine kurzsilbigen nomina mit endungslosem nominativ existierten, entstand nach analogie von seli u. a. der nom. sg. beki. Doch ist zu beachten, dass diese entwicklung nur in gewissen strichen stattgefunden hat, anderwärts wurde nach massgabe der flectierten formen ein nominativ *bekki entwickelt.

$\left.{ }^{8}\right)$ Kluge im Etym. wb. s. 54 setzt ein got. *prakkeis voraus, was nicht richtig sein kann, da die heutigen mundarten z. b. schwäb. $\bar{E}$ verlangen (schwäb. $d r e ̈ k$ ), die grundform ist vielmehr ein germ. * prekkaus *prezn- (nach $r, l, v$ ist skand. bekanntlich die brechung nicht eingetreten, vgl. Noreen, Anorw. und aisl. graw. $§ 86$ nebst nachtr.). Es 
B. Ahd. zapfo, anord. tappi, ags. tappa zapfen, s. 165; abd. chapf s. 167, schwäb. k'apf hoher punkt mit fernsicht, schweiz. kchapf Bachmann s. 36, vgl. noch Kluge, Beitr. X, 442; - ahd. hnapf, ags. hnaep (-ppes) napf; - ahd. zopf, andd. topp, ags. top (-ppes), afries. top, anord. toppr zopf; - ald. scopf, scof, scopho Holtzmann, Ad. gr. s. 309, ags. sceop s. 167. 171; - ahd. scoph, mhd. schopf, schopfe, schupfe, ags. sceoppa schuppen s. 167, Beitr. VII, 134 anm; - ahd. chrof, chropf, ags. crop (-ppes), anord. kroppr kropf s. 167.171, Beitr. VII, 134 anm.; - ahd. chnopf, chnof, tirol. knoaff (s. Dwb. s. v.), engl. knop, ndl. knop (anord. knappr, ags. cnapp, mengl. knap knopf mit auffälliger vocalstufe), dazu mbd. knouf, ndl. knoop knauf s. 167. 184.185; - ahd. topf, tof, topfo, mhd. topf, topfe

ist dafür folgendes geltend zu machen. Die bedentung des wortes scheint ursprünglich 'wertloser bodensatz' oder ähnl. gewesen zu sein, wie sie heute noch in mundartl. benennungen z. b. schmalzdreck = rïckstand des ausgeschmolzenen fettes durchscheint. Damit tritt das wort in zusammenhang mit der reichen sippe für 'hefe' und verw. Ahd. trestir, ags. darste ist bereits von Kluge, Beitr. IX, 195 aus *trahst- erklïrt (vergleiche $\mathrm{zu}$ diesem gesetz noch mndd. tester, soest. tęsto sehne zu ahd. zähi zäh Holthausen, Soest. ma. § 69) und zu engl. dregs, anord. dregg ferner zu apreuss.dragios hefe oder lat. fraces befe gezogen worden. Das vor -st geschwundene $-h$ ist in ahd. pitrohsilit Graff V, 505 bewahrt, dagegen gleichfalls in trusna, trusana, truosana etc., erdruasnita Graff V, 546 gefallen. Es ist mir nicht zweifelhaft, dass mittels labialismus ags. drabbe hefe, ahd. trebir Graff V, 481, ags. draf, ndl. draf, engl. draff, drab anzuknüpten sind. Die german. grundform wäre demnach *dhroz:*dhrez, *dhroh : *dhreh. Apreuss. dragios, abulg. droždije hefen; ir. drog, droch malus, kymr. drwg malus, corn. droch, drog malus (die sinnliche bedeutung in dem aus dem celt. entlehnten roman. droga, frz. drogue vgl. Littré) Ks. zs. 24, 202 führen anf idg. $g h$,

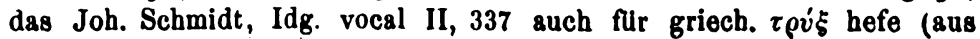
*dhrogh-) angenommen hat. Kluge hat auch bereits a. a. o. lat. fraces hefe beigezogen. Ich glaube dass es auch in formeller beziehung nicht von den bisher besprochenen auszuschliessen ist. Ich vermute, dass der nom. sg. *frax für "fraxs $(\chi=g h)$ steht und im gefolge der ubrigen nominative auf $-x=c s$ die obliquen casus und die pluralformen $c$ bekamen, zumal da intervocalisch $g h$ zu $h$ werden musste, also eine grosse differenz entstanden wäre. Möglicherweise waren auch faex, faeces und das gleichbedeutende flocces von einfluss. So liessen sich sämtliche formationen der europ. idg. zweige unter der stammform dhrogh: dhregh: dhrgh vereinigen. Anord. prekkr, ahd. drech knüpfen an ein schwach flectierendes * dhreghon an. 
kreisel s. 165 ; - ahd. stopfo, stupf punkt s. 165 ; - mhd. schrapfe werkzeug zum kratzen (aus skrotn-) gegen ahd. scrëvon einschneiden; - ahd. kroph = ags. crop; anord. kroppr haken Beitr. VII, 134 anm.; - ahd. charpfo, mhd. karpfe, md. karpe, schwăb. $k$ arpf karpfen, age. carp gegen anord. karf mit grammat. wechsel; - hierher gehört auch schweiz. hëpf hefe Winteler 8. 44, Stickelberger, Mundart von Schaffbausen 8. 22 (je mit offen $\ddot{e}$, also nicht aus a umgelautet), bair. hepfen Schmeller, Ma. 8. 93. Dieses wort steht offenbar in ablaut zu ags. haf und beruht auf einem gern. "hëbn- > *hëpp; ag8. haf setzt wurzelbetonung voraus, wie die ahd. $j$-ableitung hevo: heffo befe vgl. schwäb. hefa befen (mit geschlossen $e=$ $i$-umlaut von $a$ ); - ahd. gelf (substant.) vgl. Graff IV, 197, mhd. gelf, gelpf lärm, ubermut Mhd. wb. I, 518, ags. gilp, gealp gegen anord. gjalfr sonitus vgl. oben s. 505 das adj. gelpf; - ahd. mhd. krumpf, chrampf = anord. krappr, mhd. krimpf neben krumb, ags. crumb krumm 8. 182, Beitr. VII, 134 anm., ebenso das substant. mhd. krampf, krimpf, ahd. krampf, chrampfo, as. cramp krampf; - ahd. houf, mhd. houf, hüf, ahd. $h \bar{u} f o$, mhd. hūfe, houfe ${ }^{1}$ ), mfr. hop Beitr. I, 27, as. hop, age. heáp (vgl. abulg. kupü) haufen s. 184.

C. Ahd. scaz, got. skatts, anord. skattr, ags. sceatt, afries. sket, as. skat schatz; - ahd. hroz, roz, mbd. roz, rotz s. 167; - abd. chozzo, choz kleid, mhd. kotze, as. cot (tt), mlat. cotta (?) s. 165 ; - ags. gnaet $(t t)$, nhd. gnatze, gnitze kleine stechfliege s. 167; - mndd. snūte, ahd. schnauze Beitr. VII, 134 anm.; mhd. glanz glanz, ahd. mhd. glanz glänzend : mhd. glander glanz, glanst glanz vgl. Beitr. VII, 134 anm.; - anord. hottr, ags. hoett hut gegen ags. hôd, ahd. huot 8. 167; - ags. steort,

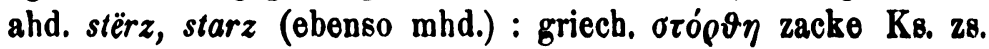
26, 92, ebenda, gleichfalls hierhergehörig, ags. flint kiesel, feuerstein, schwed. flinta, dän. flint stein : griech. $\pi \lambda i v \vartheta o ̣$ ziegel; ags. catt, anord. kottr, ahd. chazza, mhd. katze gegen westgerm. *kadazan (Kluge, Nom. stamb. § 28), ahd. chataro, mhd. kater kater, das verhăltnis der dentale ist wie Kluge bemerkt dem von an. kip : ahd. chizzi analog, wăhrend ahd. -t- auf

1) Ahd. mhd. $f$ ist wahrscheinlich verschiedener provenienz, in dem schwachflectierten nomen wol = germ. $f$ (háufo : hüzn-). 
gemination von $\delta$ vor $r$ zurtuckgeht, ist z. b. in siebenb. $k a d r$ Beitr. XII, 146 dieselbe nicht eingetreten.

D. Ahd. fol (ll), got. fulls, ags. full, as. full s. 168; weitere analoge fălle oben 8. 504 f., Kluge, Stammbildungslebre 8. 96; - ahd. wolla, got. wulla, ags. wull s. 168; - ahd. wëlla (Ks. z8. 27, 168) vgl. lit. wilnis welle s. 168; - ahd. polla (in hirnipolla), ags. bolla, afries. bolla, anord. bolli schale $=$ rundlich geformt aus *bhulna vgl. Kluge, Etym. wb. s. 34; - ahd. al (ll), got. alls, anord. allr, ags. eal (ll), as. al (ll) 8. 171. 167; - ahd. galla, ags. galla, anord. gall s. 170; - mhd. knolle, ags. cnoll hilgel (ndl. knol rube?); - ahd. fel (ll), got. fill, anord. fjall, ags. fell fell vgl. filmen bäutchen auf dem auge, griech. $\pi \dot{\varepsilon} \lambda \mu \alpha$ soble am fuss; - ahd. stollo pfosten, mhd. stolle vgl. aind. sthūna pfeiler, griech. $\sigma \tau \tilde{i} \lambda$ os Ks. zs. 27, 168.

E. Ich glaube, dass auch die doppelformen got. fairra, anord. fjarri, as. ferr, ahd. ferro, ags. feor; got. fairneis, anord. forn, as. firn, ahd. firni; - ahd. sterro (vgl. dazu Kögel, Beitr. IX, 326), as. sterro, ags. steorra, got. stairno, anord. stjarna, ahd. sterno und stark flectierend stern in diesem zusammenhang ihre erklärung finden. Ahd. firni gegen anord. forn lăsst noch am ablaut alten wechsel der accentuierung erkennen, den wir auch fur *sternō voraussetzen durfen. In den endungsbetonten formen musste die assimilation $r n>r r$ eintreten.1) - Ahd. narro narr kann derselben periode die gemination verdanken; alte $n$-ableitung sehe ich auch in got. qairrus sanft, anord. kvirr, kyrr, mbd. kürre, kirre zahm gegen ahd. quërdar, lockspeise vgl. Kluge, Stammbildungslehre nachtrag zu §93.

F. Gemeingerman. assimilation in der verbindung $-m n$ scheint vorzuliegen in got. wamm fleck, anord. wamm schande, ags. vom (-mm) flecken, as. wam, ahd. wam (Graff I, 851); ags. hvom (-mmes) winkel; - anord. dimmr, ags. dimm dunkel u. a.; - anord. hrammr pfote zu ald. rama, schw. ram; anord. gammr geier : schw. gam u. a. bei Noreen, Anorw. und aisl. gram. § 206 anm. Ob ags. assa esel im gegensatz zu

1) In den afries. rechtsquellen ist einmal (Richthofen s. 131, 12) nom. pl. stera die sterne belegt, eine form, die falls correct uberliefert wie anord. spakr etc. (8. o. s. 514) erklärt werden muss, im nom. starker flexion war -r lautgesetzlich, *ster neben *stern $\bar{\gamma}>$ * sterō. 
anord. asni urgerm. assimilation erfahren hat, ist nicht beweisbar.

Es ist bereits oben s. $508 \mathrm{f}$. davon die rede gewesen, dass Kluge auch geminierte medien und spiranten urgermanischer herkunft zulässt, dass ich aber seiner methode der erklärung nicht zustimmen kann. Für die existenz gemeingerman. geminierter medien sprechen einzig und allein eine kleine zahl nord. nomina, es sind: anord. krabbi vgl. ags. crabba krabbe s. 162.167; anord. koddi vgl. ags. codd tasche s. 162; anord. toddi, todda wolle, wollenknäuel, vgl. ndl. todde lumpen, engl. tod gewichtmass für wolle, anord. baggi neben pakki last s. 166, Beitr. X, 441; anord. vagga wiege \&. 166 ist sebr zweifelhaft vgl. Ks. 28. 24, 507 (wie ist damit ahd. wiga, mhd. wige vereinbar?), vgl. noch nfries. wedse, afries. widzia, wigge Beitr. XI, 209. Ausser betracht bleiben kann auch baggi:pakki. Für die ubrigen nehme ich an, dass sie wahrscheinlich unter die zahlreichen sludgerm. lehnwörter gehören, was keine schwierigkeit haben durfte. - Noch bleibt eine anzahl von fällen ubrig, für die ich glaube, dass bereits urgerman. doppelconsonanz (tenuis) nach Kluges regel vorhanden war, so z. b. mhd. ratze, ratz ratte. Ich stelle dieselben zurtick, da nach meiner meinung die belege fur die einzelnen worte nicht auf grund dieses éinen gesetzes gedeutet werden können, sondern nur mittels kreuzungen, die durch die westgerm. consonantendehnung vor $-n$ deutlich werden, sie folgen unten s. $529 \mathrm{ff}$.

\section{Die westgerman. consonantendehnung vor $-n$.}

Das material, wie es Klnge s. 166 gesammelt hat, ist nach unbefangener betrachtung durchaus den dehnungserscheinungen vor $i, w, r, l \mathrm{zu}$ vergleichen, es ist nicht, wie in der gemeingerm. assimilationsperiode, irgendwelche flexivische veränderung des wortes eingetreten. In den nominibus, die ich in folgenden zusammengestellt habe, liegt wechsel zwischen einfacher und doppelter, stammbafter consonanz vor, ohne dass $i, w, r, l$ nachweisbar wären.

A. Gutturale.

1. Westgerm. $z$ erscheint geminiert ags. als $z z, c z(=g g)$, as. $g g$, ahd. $g g$, dem die gleichwertige schreibung $k k$ pa- 
rallel geht; der einfache laut ist durch ags. $-z^{-}$, as. ahd. $g(k)$ vertieten.

Schwāb. mauk $\left.{ }^{\prime}\right)(\partial u=$ mbd. $\bar{u})$ obstansammlung, die sich kinder im heu versteckt anlegen, auch in ardern dialekten ublich, dazu ags. $m \bar{u} c \bar{z} a$ neben $m \bar{u} z a$ haufe, anord. múgi haufe, menge, Ks. z8. 24, 210 f.; - schwäb. roka, mhd. rocke, ahd. rokko, roggo gegen ags. ryge, anord. rugr roggen (ebenso rogn in Remscheid Beitr. X, 423) s. 16i. 176; - ags. focze füchsin mit gramma-

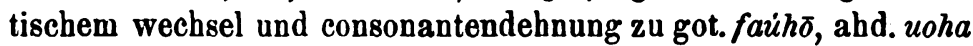
(Germ. 9, 19) s. 161; - ags. frozza, selten frocza (vgl. Sievers, Beitr. IX, 226) frosch (tiber ags, frocca, anord. fraukr s. o. s. 515) neuengl. frog 8. 161.167.176; - ags. docza, dozza hund vgl. neuengl. $d o g$ 8. 161. 166. 177; - ags. suz̧za, sucza vgl. Sievers a. a. 0. motacilla (bachstelze); - nach ir. clog glocke liegt in abl. glocka, mhd. glocke, ags. cluzze, clucge geminiertes $g$ zu grund, anord. klukka ist also lehnwort, anders Kluge, Beitr. $\mathrm{X}, 441$; - schweiz. lunko lunge Winteler s. 61, Bachmann s. 25 gegen ahd. lunga, ags. lungen s. 179; - schweiz. das junki das junge Bachmann s. 25; - schweiz. wak kieselstein Bachmann s. 20, vgl. Schmeller, Wb. II, 844 abd. waggo, wacko, mhd. wacke s. 162 ; - schweiz. mägi : mäki mohn zu ahd. mago, mhd. mage $\left.{ }^{2}\right) ;-$ schwäb. šnëk, schweiz. šnëko schnecke Bachm. 8. $22.44 ; \ddot{e}$ in diesen mundarten verbietet an $a$-umlaut $z \mathrm{zu}$ denken, es kann demnach für ahd. snëggo, mhd. snëcke, snëgge, fries. schnigge Correspondenzbl. f. ndd. sprachf. XI, 35 nicht ein

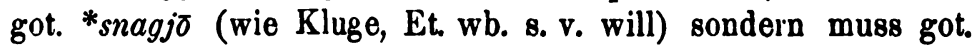
*snigo ongesetzt werden, dazu steht im ablaut ags. sncezel (mit einfacher consonanz), die $i$-stufe auch in anord. snigill; ags. sceaza : sceacza buschwald, vgl. mengl. schaze, anord. skagi, skógr wald; ahd. scahho landzunge, mhd. schache stuck einzelnen waldea, steben nit den ausserdeutschen wörtern in grammatischem wechsel und zeigen $h$-gemination, s. 158 f. 167 . 177; - hierher gehört wahrscheinlich auch ndl. hark, ndd. harke gegen engl. harrow (ags. *hearze), die skandinav. belege

1) Oberd. inl. $k$ meiner transcription ist eine hanchlose fortis als fortsetzung von westgerm. $g g(k k)$.

2) $\bar{a}$ ist in diesem worte nicht berechtigt, wie ausser den schweiz. formen schwäb. māks ō ma mohnsamen beweist, vgl. Möller, Beitr. VII, 517. 
wie anord. herf, dän. harv zeigen labialismus; - schwäb. houkə eulen, uhu, habichte $u$. ähnl. bezeichnend, das zweifellos zu ahd. hūwo, labialisiert (aus - zw-) hūfo, mhd. hūve eule gehört; nach skr. ulüka (Beitr. XII, 212) ist die grundform $(h) \bar{u} z n-$, (das wort zeigt denselben wechsel zwischen $w$ und $z$ wie ags. sāwon: sazon 1) u. a.), geminiertes $\zeta$ muss dem $k$ von schwăb. haukə zu grunde liegen; - ahd. hägo : schweiz. hōka, hāka Winteler s.61, weiteres uber das wort siehe u. s. 529; - Hierher wol auch ags. éarwicza ohrwurm (Sievers, Ags. gr. ${ }^{2} \$ 216$ anm. 2).

2. Westgerm. $-k$ - des stammes erscheint in gemination als $k k, c c$, nur hochalem. als -kch- vor alters wie heute, vgl.: ags. draca, ahd. trahho, mhd. drache aus lat. draco, griech. $\delta \rho \alpha ́ x \infty v$ zu ahd. traccho, mlid. dracke, bair. dracke vgl. Schmeller, Wb. I, 565, Ma. s. 87, DM. III, 16; s. 0. s. 510; - schwăb. baoka trommeln gegen ags. beacen = ahd. bouhhan signum, dazu wahrscheinlich mit ablaut mhd. būke pauke s. 0. s. 510; - ebenso bair. zagng zeichen Schmeller, Ma. \$. 85 gegen ags. tacn = ald. zeihhan $\left.{ }^{2}\right)$ u. a.; - ahd. lahha (laccha), mhd. lache pfutze ist im bair. als lachen neben lacken bewahrt, vgl. Schmeller,

2) -z- für - $z^{w}$ - war vor folgendem $-u$ - lautgesetzlich; $w$ in ws. sāwon wird aus dem part. prät. stammen, vgl. ags. serven, zeseowen, während ags. ziseozen nach sazon, ebenso northumbr. part. zesezen, praet. se zun, von denen, wenn auch spät bezeugt, eines gewiss die alte bildung zeigt und zwar sebe ich diese in zesewen, zeseowen ( $w$ aus $-z w$ nach Sievers gesetz). saezon knlipft an ein germ. *sazum aus *sazroum an, $w$ ist vor eintritt des Sievers'schen gesetzes gefallen. Die beziehung zwischen praet. und part. praet. zeigt deutlich part. zesáwen zu pruet. sáwon, Sievers, Ags. gr. ${ }^{2} \$ \S$ 73. 391, 2. Das alter der $-g$-formen wird sehr gewichtig auch durch die continentalen mnl. saghen, mfränk. sägen, conj. sege z. b. in König Rother bezeugt, Paul, Mhd. gram. $\$ 162$, anm. 2, Beitr. VI, $541 \mathrm{ff}$., Osthoff, Beitr. VIII, $262 \mathrm{ff}$.

2) Dieses beispiel halte icb für durchaus gesichert, trotz bair. ming-ga München; dieses kann nur aus einem dativ. plural. "münken (nicht *münchen) entstanden sein. Lat. monachus ist bekanntlich als monacus aufgenommen, wie zweifellos ags. munuc, afries. munik; obd. $k$ in diesem ortsnamen beweist, dass wir hier, wie in einigen andern wörtern, vgl. z. b. ahd. storah : neualem. stork neben ahd. munih ein *munc(vgl. anord. munkr) vorauszusetzen haben (ebenso ein *munt- $>$ münze neben ahd. munizza - moneta?), in letzterem ist regelrecht auf diesem gebiete die'verschiebung nicht eingetreten. 
Wb. II, 435, Ma. s. 86. 87, DM. III, 306; Weinhold, Bair. gr. s. 187, zu letrterer form ital. lacca tiefer grund vgl. Kluge, Et. wb. s. 190, Beitr. IX, 166; - ahd. broccho, mhd. brocke, gegen got. gabruka Kluge s. 155, eiegerl. bro ${ }^{a}$ cke Heinzerling \&. 102, schweiz. brckcha Winteler 8. 61 ; Bachmann a. a. 0. 8. 40 hat die doublette brokcho : brocha bewahrt; - siegerl. mocke weibliches schwein Heinzerling a. a. 0., schweiz. mokcha Winteler s. 61 , Bachmann 8. 48, vgl. mhd. mocke klumpen, brocken; dies die grundbedeutung, woraus die siegerl. abgeteilet; im schwäb. mokl kindername fur kuh, schweiz. mokchli dicker, kurzer mensch, thurg. muchel, bei Bachmann 8. 49 noch eine reihe von formen mit inl. $g$, die fur mokche möglicherweise germ. gemination verlangen; ganz analog nhd. dial. hocke (oberd. ndd.) heuhaufe' gegen oberelsãss. hü chel (= mbd. hüchel) Beitr. XI, 562 anm.; - ags. veoce, ahd. wiohha, wioh, mhd. wieche docht, mit gemination ags. vecce, mhd. wicke, siegerl. wēke, bei Vilmar idiot. \&. 454 wīke, wicke, ndd. wocke, wucken docht vgl. DM. II, 512, 19; - ahd. bahho : baccho, ebenso schweiz. bacho : bakchə Bachmann s. 40. 51, bei Winteler 8.61. Bachmann 8. 51 baka ebenso schwäb. baka $(k=k c h)$, vgl. as. kinnibako, ndl. kinnebakken, ahd. chinnibahho: chinnibaccho Kl. s. 166, 167; - ebenso wol ahd. braccho hund, vgl. Winteler 8. 61 , Kluge s. 165 ; - ahd. stëhho : stëccho, mbd. stëcke, stëche stecken, dazu schwäb. štęaka, vgl. noch ags. sticca 8. 166; - mhd. zëche : zëcke, ersteres bei Winteler s. 52, letzteres in schwåb. zek' zecke, vgl. engl. tike : tick s. 166; - schweiz. krāk ( $k=k k$ in Kerenzen) krähe Winteler 8. 62 zu anord. kwáka1) krähe; - schwåb. šnōko schnaken, mhd. snāke, anord. snákr 8. 179 (möglicherweise - $k k$ - gemeingerman.), Beitr. VII, 133; ahd. sluko consumptor $\mathrm{Ra} 123,31=$ sluhho $\mathrm{gl}, \mathrm{K}, \mathrm{Pa}$, bei Kögel, Ueb. d. kerno, gl! 8. 89; - Bachmann 8. 40 verzeichent noch:

1) Ich glanbe dass anch in anord. kráka selbst $k$ ans $k k=z^{n-}$ entstanden ist. Aus dem Anhaltischen in der nähe von Dessan kenne ich krahge krähe d. i. kräze, nimmt man dazu mhd. krärve, ahd. chräwa, ags. cräve krähe so verhslten sich $w: \zeta$ ganz wie *hüvo zu schwäb. houks: s. 8. 522. Bereits urgerw. ist $-z^{n-2 u}-k k$ - assimiliert resp. verschoben in anord. kráka; schweiz. $k$ kann demgemäss wie $k$ in schwäb. houks auf westgerm. - $3 z$ - beruhen. V'ilmar idiot. $8.222 \mathrm{krăke}$ rabe, neben kracke sind wol mit sicherheit fitr urgerm. geminate (wie anord. kräka und möglicherweise schweiz. $k r \bar{a} k$ ) geltend zu machen. 
balcha : balkcha balken, mhd. balke, ahd. balko, vgl. ags. bealca; - wulcha, wula, wola : wulkcha wolke, ahd. wolchd neben wolcan, ags. volcen neben wolc vgl. Beitr. IX, 216'); - schweiz. aøkcha neben ächa butter vgl. DM VII, 334. 346, ahd. ancho, mhd. anke; - hierher wol auch ags. hracca nacken : ags. hraca kehle - ahd, rahho rachen.

3. Westgerm. $h$ erscheint in der gemination als $h h$, heute inlautend zwischen vocal $=c h$, wăhrend der einfache laut verstummt ist: ags. pruh, anord. pró trube, mhd. truhe, gegen schwäb. druch truhe, weiteres thber die worte s. u. s. 529; bair. tâhha : tâa thon Schmeller, Mla. s. 100, ahd. dāha, mhd. tāhe, got. pāaho, ags. pó, anord. pá thou; - bair. zecho zehn Schmeller a. a. 0., schweiz. zecha, zechnar Bachmann 8. 14 (hier ist -ch- aus den flectierten formen von ahd. zëhan herzuleiten); - bair. zēcha Schmeller a. a. 0., schweiz. zēcha zehe Bachmann 8. 14, ahd. zèha, mhd. zēhe u. s. w., vgl. Ks. zs. 24, 442; - ahd. scahho, mhd. schache gegen ags. sceaza 8. o. \$. 521; - ebenso ahd. zuhha : zuga runzel Kluge s. 159; - aus Bachmann 8. 14 gehören noch hierher: schweiz. blacha : blaho, mhd. blahe decke; - schlecha : ahd. slēha schlehe ${ }^{2}$ ); - schweiz. chuecho schlittenkufe, abd. choho; - gehören hierher ags. crohha luteum, ags. scocha lenocinium, vgl. Beitr. IX, 227?, als geminiertes $h$ h muss auch ch in md. fache oft, facher öfter 8. 0. 8. 515 erklärt werden, oder liegt $k$ aus germ. $k k$ reduciert zu grund?

\section{B. Labiale.}

a) Wechsel zwischen einfachem and geminiertem westg. $p$ in ahd. stapfo : staffo stapfe s. 166 ; - ahd. tropfo : troffo tropfen, vgl. ags. dropa, as. dropo, anord. dropi, ripuar. droppe Beitr. I, 26 neben droffe Beitr. IX, 380, nfries. druppah Correspondenzbl. f. ndd. spr. XI, 34, siegerl. droabbe Heinzerling 8. 77,

1) Die hier von Sievers gegebene erklärung von ags. wolc befriedigt mich nicht, schon weil ahd. wolcha daneben besteht; ich halte es für sehr wahrscheinlich, dass ags. wolc wie ahd. roolcha $(c h=c c h$ ?) urgerm. geminste aus $-k n$ - bei endungabetonung enthalten (s. Kluge, Etym. wb. 8. จ. volkno-).

9) Indessen kann hier die gemination auch durch $-n$ - bewirkt sein, vgl. Beitr. VII, 440, wie dies für zeche, sigerl. zache, ags. teohhe, teohhian vgl. Kluge s. 158 , ags. seohhe, zeneahhe Kluge s. 158 zu gelten hat. 
Kluge s. $166 ;-$ mhd. schapfe, schwăb. šapf : mhd. schaffe, ahd. scaffo schöpfgefäss; - ahd. wituhopfo, widehoppa Germ. 9, 20 : nituhoffo wiedehopf s. 166; - ahd. gapfa : gaffa mitra s. 166, vgl. Holtzmann, Ad. gr. s. 309; - ahd. pfroffo : *pfropfo setzling, pfropfen, vgl. probur Rc Beitr. IX, 312; - ahd. chiffa : chipfa humerulus Graff IV, 370, Kluge s. 166; - ahd. goffa : gopfa Graff IV, 176, Holtzmann a. a. o., vgl. mlid. gupfe, schweiz. gupfo aufsatz am ofen Winteler 8. 44; - ahd. gislāpfa nupta Graff VI, 802, Weinhold, Al. gr. s. 123 , Kluge s. 178; - ahd. chrāpfo, schwäb. krapfo krapfen : ahd. chrāfo s. 178. 180, Beitr. VII, 123; - ahd. snëpfo, mhd. snëpfe gegen mittelengl. snīpe schnepfe; - mhd. harpfe, ahd. harpfa, bair. scbweiz. harpfo bei Schmeller, Ma. 8.93, Winteler s. 43 u. a. harfe vgl. ags. hearpe; - ahd. hëlpra hilfe, vgl. Kögel, Ker. gl. s. 72, Otfrid III, 10, 37 (V) neben hilfe, hëlfe u. a.; - ahd. seipfa, schwãb. soapf seife : ahd. seifa, mhd. seife, ags. sâpe, wie die form sâpo bei Plinius beweist, bestanden im german. auch formen ohne $j$-ableitung ${ }^{1}$ ), vgl. Ks. zs. 24, 436; - ahd. hopfo, mhd. hopfe, schwăb. hopfo u. a. hopfen, ndl. hoppe, mittelengl. hoppe, vgl. mlat. hupa s. 0. 8. 510. Ob ags. doppa, stoppa, sceoppa, loppe, glappe, vgl. Kluge 8. 165. 172 , hierher gehören, ist nicht $\mathrm{zu}$ entscheiden.

b) Westgerm. $\delta$ erscheint geminiert ags. als $b b$, as. $b b$, ahd. $b b, p p$ (nur variationen der schreibung, wie bei $g g, k k$ ); in den heutigen dialekten scheint allgemein eine hauchlose fortis, doch mit landschaftlich wechselnder intensităt zu entsprechen: siegerl. abbe Heinzerling s. 73, ndd. obbe grossvater neben siegerl. āwe, hess. ābe grossvater, vgl. anord. af grossvater, got. aba mann; - ahd. rabo: rappo, mhd. rabe : rappe rabe, ersteres in der schriftdeutschen form, letzteres in schwab. $k r a p$ ( $k$ - prăf. ge-), schweiz. rap Winteler s. 59, Kluge s. 162. 166, die von Holthausen, Ks. z8. 27, 623 vorausgesetzte grundform idg. *kropnós ist in dieser form nicht zulässig, ihr müsste notwendig germ. *hrappa- entsprechen; - ahd. chnabo : chnappo, mhd. knabe : knappe, dieselbe doublette in der schriftsprache mit bekannter bedeutungsdifferenzierung; uber ags. cnapa s. u. s. 529 f. 532; - ahd. scuoppa, mhd. schuoppe, schwăb. šuəpə, mhd.

1) Auch im finn.-lappischen belegt Thomsen-Sievers 8. 68 saipo neben saippio, saipua, saippua. 
schuope, ndd. schob schuppe Kluge 8. 179; - ahd. kluppa, chlubba, mhd. kluppe zange Beitr. VII, 134 anm., Kluge s. 162. 166, der einfache consonaut in ald. chlobo, mhd. klobe, andd. klobo, anord. klof fessel; - ags. drabbe hefe, gegen ags. draef hefe, abd. trebir Graff V, 481, vgl. oben 8. 516 f. anm., Kluge 8. 162; - siegerl. globb Heinzerling 8. 74, dichter haufe, gegen engl. club vgl. Kluge, Etym. wb. unter 'kolben'; - schwäb. hō $p^{c}=$ mhd. hāppe, ahd. hāppa hippe zu westgerm. *hēta (vgl. griech.

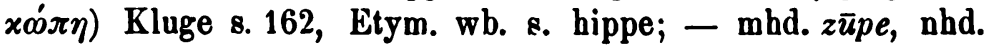
zaupe hlindin 8. Kluge s. 178 anm., bei Vilmar, idiot. s. $478 \mathrm{f}$. mit ablaut zopp, der einfache laut in hess. ziwwe, ndd. têwe, mndl. tëve, vgl. noch Ks. 2s. 24, 429. 464 woselbst sich ergibt, dass der labial auf altem velar beruht, vgl. ahd. zoha Germ. 9, 19; - dem ablautsverhăltnis zwischen mbd. zūpe : hess., zopp entspricht genau schwäb. naupe (nach Lexer, D. wörterb. s. v.): kärnt. noppe, (md. nuppe, noppe, ndd. noppe) ursprunglich knoten in der wolle, dann launen, grillen; - schweiz. chnupa knäuel Winteler 8. 59, Kluge s. 178 anm. : mhd. knübel; - mhd. tāpe = schwãb. $d g^{-} p$ pfote Kluge s. 179; - alsd. rūpa : rūppa, mhd. rūpe : rūppe, ndd. rüpen, siebenb. rop Beitr. XII,144, raupe Kluge s.179, wo auch nhd. graupe angezogen wird; - bair. grueppen gruben Schmeller Ma. s. 62; trauppen trauben s. 82, vgl. ahd. truppo : trubo Kluge 8. 166; - drappo lex. Alem. III, 5, Germ. 9, 21 : ahd. trabo Graff V, 480 vgl. noch Diez, Etym. wörterb. ${ }^{3}$ s. 158; - hierber mögen ferner nach Kluge ahd. trappo 8. 162. 177; ags. ebba (: got. ibuks) 8. 162. 167. 177; ags. pl. sceabbas rabies s. 161 (doch Leo 57 nur sceabus dagegen sceabbed schăbig), lobbe, scrobb s. 162 gehören, ich stelle auch ags. crabba (: ahd. chrebiz krebs) krabbe hierher, uber anord. krabbi s. 0. 8. 520.

c) Westgerm. $f$ ist geminiert durch $f f$ vertreten $\left.{ }^{1}\right)$ : ahd. laffa : got. lofa, anord. lofi hand vgl. Sievers, Beitr. V, 530, Winteler 8. 43, Kluge 8. 159; - mhd. schroffe : schrove felsklippe Kluge s. 159. 167, bei Schade, Ad. wb. s. v. bair. schroffen, schweiz. schrofen; - ags. snoffa schnupfen Kluge 8. 159 ; - schweiz. wäffo, schwăb. wöfo $(f$ kann $=f f$

1) Auf die von Kluge s. 159 angezogene müglichkeit, ob altes ff hd. nicht zu pf geworden sei, komme ich demnächst ln anderem zusammenhang zurlick, wo sie sich als unberechtigt erweisen wird. 
sein) wappen, neben schweiz. wäpa Winteler s. 58 wappen ${ }^{2}$ ), waffe. Nach massgabe von griech. ö $\pi \lambda o v$ ist ein german. stamm $w \bar{e} f: w \bar{e} t$ anzusetzen, deren westgerm. $n$-geminationen in den obd. mundartlichen belegen sich reflectieren. Mfränk. wäpen Beitr. I, 23, ags. woepn, anord. vápn, vọpn, got. vêpna sind auf gemeingerm. assinilation von $\delta n>p p$ zurluckzufuhren, $n$ - ist aus den nichtsynkopierenden oder stammbetonten casus bervahrt und -pp- nach dem langen sonanten vereinfacht, vgl. s. 530 .

\section{Dentale.}

a) Westgerm. $-t$ - des stammes ergibt im geminationsfalle hd. einen wechsel $z$ wischen $t z: s$, ahd. $z: z z$. Da nun aber in diesem puncte die schreibungen ahd. periode durchaus unzuverlăssig sind, von einzelnen normierten gebrauchsweisen abgesehen, haben die heutigen dialekte den ausschlag zu geben: schweiz. šprotza : schriftd. sprosse Winteler 8. 173, vgl. Kluge 8. 166; ahd. sprozzo, mhd. sprozze entsprechen ags. sprota, anord. sproti sprosse unmittelbar $\left.{ }^{2}\right)$; - hess. strotte, strotze luftröbre Vilmar, Idiot. s. 404 zu mhd. strozze Mhd. wb. II, 2, 698; mndl. storte, strote kehle, afries. strot in strotbolla kehlkopf, gurgel Richthofen, Wörterb. 8. 1054; - mhd. gieze, ahd. giozo : schweiz. giassa Winteler 8. 171.173; - vielleicht auch mhd. ganze : ganze, abd. ganazzo gănserich, vgl. ags. ganot; - schwăb. gqotsə pflughandhabe : elsäss. keis Strassburger studien II, 168, vgl. J. Grimm, Geschichte d. deutschen spr. s. 57; Graff IV, 287 hat die schreibungen gaiza, keizza, Paul, Beitr. VII, 121 setzt $i$-bildung an; - ahd. schnautze : ndd. snüte, mengl. snoute bei Paul, Beitr. VII, 134, ahd. snuzza = mnd. snüte, weiteres s. u. 8. 530; - Lierher gehört wol auch hess. trotze mistjauche, s. Vilmar, Idiotikon s. v.; - aus dem ags. ist mir kein sicheres beispiel bekannt, doch glaube ich in dem nach Sievers rhyth. mischen regeln (vgl. Beitr. X, 312) unmōglichen vers Beów. 1027: fyr scotenum, am einfachsten scottenum (typus C, Beitr. $X, 297,5)$ zu emendieren, die schreibung eines einfachen statt

1) Diese form mit kurzem $a$ (ablant zn $\varepsilon$ ?) ist, so viel ich weiss, nicht obd.-dialektisch nachweisbar.

9) Im siebenblirgischen lautet das wort heute špruss : špros, nach der analogie von ílots, kann letzteres auf *šprōts beruhen und $s$ von der nebenform eingedrungen sein, vgl. oben s. 131. 
doppelten -t- ist bekanntlich nicht selten, vgl. z. b. vitig 1056 oder hetendum aus der Elene 18, vgl. Beitr. X, 518; scottenum misste aus *scottnum analogisch umgebildet sein. ${ }^{1}$ )

b) Westgerm. $d$ erscheint im hochd. geminiert als $d d=t t$, womit die gemination von westgerm. $b$ zusammengefallen ist, der lautwert ist heute hauchlose fortis: siegerl. ratt unkraut unter weizen und roggen, Heinzerling 8. 109, dazu ahd. raddo, ratto : rado, rato Graff II, 470, andd. rada, mhd. raden, rate, ratte, raten, ratten, nhd. raden, Kluge s. 167 ; - schwäb. krott krōte zu ahd. krota, krëta krōte; - siegerl. sodde pfütze Heinzerling s. 121 neben sorrel $(r r=d)$ mistjauche, hess. sotte, sutte Vilmar s. 389, mhd. sute, sutte, sudde vgl. besudeln; - mhd. slite : slitte schlitten, anord. sleði, die gemination wird bewiesen durch ital. slitta, dann durch den quantitätswechsel in heutigen fränk. dialekten, aus denen ich šlīda : šlita kenne; - ahd. zotto : zota, mbd. zotte : zote, ndl. todde und das sicher entlehnte anord. toddi (s. 0. 8. 520), vgl. engl. tod; - ahd. tutta : tuta, mhd. tutte : tute(?) brustwarze s. 166; - ahd. chlëtta, mhd. klëtte : mhd. klëte, ahd. chlëta, ags. clipe klette s. u. s. 530; - zweifelhaft ist siegerl. schadde schatten Heinzerling 8. 125, mhd. schate, ahd. scató, as. scado, da möglicherweise $w$-gemination zu grunde liegt; - hierher gehören wol noch: ags. budda kăfer Kluge 8. 162. 166. 177; westfăl. hodde geronnene milch Jellinghaus, Ravensb. ma. s. $56=$ hess. hotten Vilmar, Idiot. 8. 176; ahd. scotto schotte, geschlagene mileh Grimm, Gesch. d. d. spr. s. 1007; - ags. sceadd maifisch, codd (= an. koddi) tasche (?) 8. 162 ; - ahd. gibrëtto, $-a$ brett 8. 166 ?

d) Geminiertes $s$ liegt vor in ahd. meissa : meisa meise Graff II, 874, schweiz. meissə Winteler 8. 45, vgl. ag8. māse, anord. meisingr.

1) Ueber die stelle handelt Kluge, Beitr. VIII, 533. Ebenda werden genannt: nefenum zu nefa Haupts zs. IX, 485, L'otenum Beów. 903. Die alte casusendung erscheint in oxnum, worðiznum, beonum, flänum die Kluge ebenda beizieht. Ich komme unten $8.545 \mathrm{f}$. darauf zurïck, und bin der meinung, dass, wie auch Kluge andeutet, Eotenum nach gen. pl. Eotena ebenso oxenum nach oxena (nach einer mitteilung von Sievers erst aus jüngerer zeit belegbar, älter oxna) u. s. w. gebildet worden sind. Der gen. pl. oxena kann möglicherweise nach muster von nom. pl. oexen, exen entstanden sein und entsprechende nominative mïssen auch sonst in der $n$-declination existiert haben. 
D. Geminiertes -l-finde ich in ahd. scolla, mhd. scholle, gegen as. scola, ags. scolu scholle; - mengl. molle, mhd. molle : mhd. mol, ahd. mol molch, eidechse ${ }^{1}$ ); - schweiz. nola : nolla hügel, ahd hnol, hnollo Bachmann s. 11.

E. Geminiertes -r- zeigt mhd. knorre neben knūre knorre, mengl. knarre, vgl. ahd. chniurig.

F. Einfaches neben geminiertem $m$ ist nachweisbar in mhd. schram, schramme, ndl. schram, anord. skráma wunde, vgl. mhd. schramen aufreissen, siebenbürg. šrom weist gleichfalls auf $-m m$, s. oben s. 126.

Nun treten aber eine reihe von geminationen auf, die sich nicht ohne weiteres in die beiden behandelten perioden einreiben lassen. Es sind folgende fălle:

Ahd. truccha : ags. prūh truhe, anord. pró, mlat. trucca, schwăb. druch, 8. o. s. 524. - Ahd. chrāgo, chrāko : anord. krókr, krákr baken Kluge s. 180. - Schwäb. hōko, schweiz. hāka, hōkə : anord. háki, ags. haca, hōc haken Beitr. VII, 132 anm., 134 anm., Kluge s. 180 , 8. 0. 8. 522. - Ags. dry ze, siegerl. drijje (Heinzerling s. 89) u. a. zu as. drucno, schweiz. trochcho Winteler s. 52, Bachmann 8. 40, schwäb. druka, ahd. truckin, trokken. - Ahd. chruog, mhd. kruoc, ags. croz (crōh vgl. Sievers, Ags. gr. $\left.{ }^{2} \S 214,1\right)$ : ags. crūce, as. krūka, mhd. krüche ( $k=k k$ aus $-z^{n-}$ ), vgl. ags. crocca, mengl. crokke, anord. krukka, gegen ags. crohha (afries. krocha?) krug mit grammatischem wechsel. Wie ist aber $\bar{u} z u$ erklăren, vgl. Kluge, Beitr. X, 442? - Ags. scucca, sceocca teufel, verfuhrer: ags. scohha lenocinium (Kluge s. 165). - Ags. frocca, anord. fraukr : ags. frozza, neuengl. frog frosch 8. 0. 8. 521.

Ahd. lappa, ags. lappa, seltener loppa, afries. lappa, anord. lappi lappen Kluge 8. 165. 177. - Ags. trceppe, ahd. trappa falle, schlinge Kluge s. 165 f. - Abd. chrāfo : chrāpfo dazu obd. krāpe Kluge s. 180. - Anord. snoppa schnupfen, mhd. snupfe, siegerl. schnoappe Heinzerl. 8. 77 : ags. snoffa, schwab. s̀nupat, nhd. schnuppe schnupfen. - Ahd. chnabo : chnappo, ags. cnapa : cnafa, afries. knapa : knappa, as. knapo,

1) molle aus *molo ist offenbar eine bildung wie ahd. boro (Kluge, Nom. stammbildungsl. § 15) zu ahd. malan. 
anord. knapi Kluge s. 166. 176. - Schweiz. wāpa : wāffo, ags. wä pn, afries. wēpin (dat. sg. wēpne), anord. vápn, got. vēpna waffe (wappen) s. o. s. 526 f. - Schwäb. štompa, schweiz. štumpa Winteler s. 59 gegen stumpf, ahd. mhd. stumpf, vgl. anord. stúfr, ahd. stumbal, stumbolon s. 182. - Ahd. scoph, mhd. schopf, schopfe, schupfe : nhd. schuppen 8. o. 8. 517. - Ahd. snoppa schnauze : mnd. snoppe, mhd. snupfe Beitr. VII, 134. - Ags. *cnobba, mengl. knobbe, engl. knob knopf, nhd. knubbe, knuppe knoten, knolle gegen ahd. chnopf, chnof etc., vgl. noch mhd. knübel knöchel.

Ahd. chnodo, chnoto, chnotto, age. cnotta, anord. knütr (úknýttir), mhd. knotze, anord. knottr, dial. knotta, schweiz. chnodo Winteler s. 173, vgl. Osthoff, Beitr. VIII, 299 f. anm. Ahd. snuzza = mnd. snüte, nhd. schnautze zu ahd. snūden, snūda, snūder vgl. Beitr. VII, 134 anm., siegerl. schnudde, Vilmar, Idiot. s. 365 hess. schnūte (aber schnitze schneuzen), afries. snotte nasenschleim. - Ahd. chratto, ags. cart, crat, ahd. krëzzo, mhd. kratte, krëzze, schweiz. chratə Winteler s. 171. 173, schwäb. kręto korb. - Ahd. rato, ratta, mhd. ratte, rat, rate; ratz, ratze, andd. ratta, ags. rat, dăn. rotte, siegerl. radde Heinzerling 8. 122 (gael, radan) ratte, Klnge 8. 160. - Got. atta, bd. dial. ätte nebst spielarten, mhd. Etzel, s. 160 anm. - Ahd. latta, ladda, mhd. late, latte, ndl. lat, ags. laetta $=$ siebenb. lats Beitr. XII, 144 latte, mengl. lappe, Kluge s. 160. - Mhd. mate, matte, matze, ags. meadu, afries. mede matte, wiese. Ahd. chloz, mhd. kloz, engl. cleat (isl. klót?) kloss, mbd. klotz, engl. clot, siegerl. gludde klumpen, hess. klute, klaute klumpen Vilmar, Idiotikon 8. 209. - Ahd. chlëtta, chlëta : ags. clipe, clāte klette, nach dem Dwb. 8. v. chledwurz, ostfries. kladde, aengl. clete, clote, ongl. clotbur, brandenburg. klitz, in Sachsen klëlte und kloete Kluge s. 160. - Ahd. glat glatt, afries. gled glatt, vgl. lith. glodas glatt : mhd. glatz kablkopf, glatze = glatte stelle, vgl. Beitr. VII, 134 anm. - Ags. moppe, mhd. motte, anord. motti Kluge s. 160, mhd. matte (ags. mohpe) motte, damit verwant ahd. mado, mhd. made, ags. maba, got. maba made. -

Ags. reohha, rohha, dän. rocke, schwed. rokka, ndl. roch, rochen Kluge 8. 161. - Ags. pohha, poca, pocc, bair. pfoche pocke Kluge s. 158. - 
Kluge hat a. a. o. s. 160 f. aufgestellt, dass im anord. und ahd. $b j$ zu $t t$ (anord. motti, mhd. motte gegen ags. mobpe) geworden sei, entsprechend sollte anord. $k k$ (dän. rocke $=$ ags. reohha) auf hh zurltckzuftubren sein. Ish habe bereits oben s. 508 f. betont, dass diese geminierten tonlosen spiranten nicht mit Kluges theorie von der endungsbetonung in einklang zu bringen sind. S. 177 låsst or fur einzelne fälle offen, sie könnten der westgerm. consonantendehnung zugehören, ohne aber die möglichkeit urgerman. gemination fur reohha, pohha, snoffa, schroffe, laffa etc. abzuweisen. Es ist nicht anders denkbar, als dass Kluge in derselben weise wie bei chnabo : chnappo (s. o. s. 508 f.) eine associativisch-analogische gemination sich construiert hat, wobei die regelrecht geminierten verschlusslaute eine gemination der reibelaute veranlasst hätten.

Die geminierten reibelaute sind dem ostgerman. fremd, die skandinavischen dialekte, wie das got. weisen in den fraglichen făllen, durchaus Kluges regel adåquat, geminierte tenuis auf und so meine ich, werden wir schon durch diesen sachbestand darauf geflurt, die geminierten spiranten der westgerm. consonantendehnung zuzuweisen.

Es ist bekannt, dass die schwache nominalflexion ursprunglich wechselnde betonung, wurzelbetonte casus neben endungsbetonten besass; ich kommo darauf, so wio auf den damit zusammenhängenden ablaut der stammsilben zurtack. Die ostgerm. belege mit geminierter tenuis entsprechen der assimilation der tönenden reibelaute bei endungsbetonung, daneben haben ursprunglich nicht geminierte stimmlose reibelaute bei wurzelbetonung bestanden, die betr. nomina sind nicht in die starke flexion ubergetreten, die dehnung des stammauslauts ist erst westgerm. erfolgt. Ein nomen von der (schematischen) form $a p \bar{o}$, erscheint im paradigma stammhaft als $a f-: a t$-, in den casus, in denen bei endungsbetonung $-n$ - an den reibelaut $z u$ steben $k a m$, musste $a t n>a b b>a p p$ werden, und der ubertritt in die starke flexion lag nahe. Daneben blieb aber die schwache flexion der af- (möglicherweise auch ab-) formen bestehen: nom. sg. appa-z, afo ( $a \bar{b} \bar{o})$. Ich habe oben s. $512 \mathrm{ff}$. zu beweisen gesucht, dass appa-z (einzelsprachlich) zu $a p-(z)$ werden musste, 
die gemination nur vor vocalischer endung existierte. So erbalten wir zwei den rein lautlichen bedingungen conforme paradigmen: 1. nom. sg. ap-(z), gen. app-es, 2. nom. sg. afo $(a b \bar{b})$. Die einzelnen nomina dieser beiden gruppen verknupfte sowol die lautliche verwantschaft des stammes wie die identităt der bedeutung und so waren allseitige kreuzungen möglich. Beinahe alle möglichkeiten der combination sind in dem historisch vorliegenden sprachmaterial vertreten. Z. b. knabe, knappe: die vor der assimilationsperiode zu erschliessenden formen sind *gnabo: *gnafo, aus ersterer entwickelt sich *knapp-, bei starker flexion nom. sg. *knap-(z), gen. *knapp-es; germ. *knafo- setzt sich direct in ags. cnafa fort, wie abd. chnabo germ. *cnabo vertritt, dessen westgerman. geminationsform ist ahd. chnappo, mhd. knappe; den ursprtinglich stark flectierenden nom. sg. *knap-(z) (gen. *knappes oder analogisch *knap-es etc.) finde ich bei ubertritt in die schwache flexion in agz. cnapa, afries. knapa (dazu knappa), anord. knapi ${ }^{1}$ ), as. knapo wider. Andere fälle haben anderes schicksal gehabt: anord. lappi, ags. lappa, loeppa lappen vertreten ein gemeingerman. labn-> lapp- mit beibehaltung der schwachen flexion, wăhrend ahd. lappa an germ. *la ${ }^{*} \bar{o}$ anknupft, das erst westgerm. dehnung zu $b b>p p$ erfabren hat. Anord. krákr, krókr haken stellen urgerm. assimilation mit kurzung nach langem sonanten bei starker flexion dar, während ahd. chrāgo (chrāko) mit chnabo auf derselben linie steht. Ags. haca haken beurteile ich genau wie anord. knapi, ags. cnapa aus einem stark flectierenden *hac- $(z)$, das in derselben weise urgerm. assimilation erfahren hatte, wie die ablautsformen ags. hoc, ndl. hoek, köln. höch, hess. hoch Vilmar s. 172, wăhrend die hochdeutschen bildungen ahd. hāko, hāggo, mhd. hāke, hāgge, sohwäb. họa, schweiz. hōka, hāka auf einem westgerm. geminierten *hēzoberuhen. Complicierter ist 'trocken'. Nach ausweis von ags. drýze, ndl. droog, andd. "drōgi (vgl. Holthausen, Soester ma. $\$ 77$, a nachtr.), siegerl. drijje Heinzerling 8. 89, ndd. drēge entsprechend auch im mfränk., Schlesien, kgr. Sachsen u. a., liegt stammbaftes $-z-$ zu grund, vgl. auch Joh. Schmidt, Idg. vocal. II, 337; was die vocalabstufung anbetrifft, so hat bereits Holt-

1) Doch wol aus dem ags. entlehnt. 
bausen a. a. o. als grundformen urgerm. *drauzi-z, *drūzi-z, die tiefstufe $u$ steckt in trocken. Letzteres berechtigt urgerm. eine participiale -no-ableitung: *jruzno-, *jruzono-, *jruzeno(suffixablaut ahd. -an, -in vgl. Graff V, 512) vorauszusetzen, deren erstere form ein stammhaftes *drukk, *drokk- ergab. Dieses ist nicht mehr nachweisbar, wol aber die mischbildung ahd. truckin, trockan, deren ableitung die von germ. *druzeno-, *druzono- ist, nach deren analogie *drukk- umgebildet wurde; so erkläre ich ausser den ahd. formen auch mhd. trucken, trocken, schwäb. trukə trocken, und danach ist ein westgerm. *drokkan-, *drukkin- anzusetzen. Nach massgabe der synkopierungsgesetze musste beispielsweise das adv. *drokknō-, *drukkno- lauten, vor consonanz die geminate vereinfacht werden (s. o. s. $512 \mathrm{ff}$.) und so entstand as. drucno, drocno, vgl. auch as. drucnida. Auch von den flectierten casus aus konnte eine stammform *drukn-, *drokn- $>$ *drukin-, *drokan- entstehen, denen genau mhd. truchen trocken und schweiz. trochcho trocken entsprechen, doch kann -ch- auch auf germ. $h$ (in grammat. wechsel zu - $z$ ) beruhen, das westgerm. n-dehnung erfahren hätte. Diese letztere annahme ist nur statthaft für ahd. firtohhan : tougan occultus Beitr. IX, 181'1); schwäb. drucha truhe, vgl. ags. príh, anord. pró, urgerman. assimlation aus $-z^{n-}$ - steckt dagegen in ahd. truccha, schweiz. trukcha, ebenso truka DM. IV, 19, Weinhold, Al. gr. 8. 178. Ein germ. knujō- knoten ergab von den vocalstufen abgesehen, in der flexion einen wechsel zwischen *knupo- : *knuðo-, von diesem gieng die in anord. knútr, úknýttir, ags. cnotta, mhd. knotze vorliegende assimilation aus, während die alte stammbetonte form in ahd. chnodo, schweiz. chnodə, Winteler s. 173 erscheint, auf dehnung vor $n$ zu $p p$ weist dial. knotte. Ganz ebenso liegen die dinge bei ratte: ${ }^{*}$ rajn $>{ }^{*}$ ratt $=$ ags. rat, dän. rotte, andd. ratta, whd. ratz ${ }^{2}$ ), ratze; *rapo (*raðo) ergeben mit westgerm. dehnung mhd. ratte, siegerl. radde etc., ganz ebenso ags. laetta $=$ sicbenb. lats latte gegen ahd. ladda, latta und nicht anders sind die formen fur korb ags. craet, caert = ahd. krëzzo (ablaut), mhd. krëtze gegen schweiz. chrato : ahd.

1) Kluge setzt wechsel der auslautenden consonanz zwischen idg. media und tenuis an, was ich möglichst vermeiden m̋̈chte.

2) Das masculine genus finde ich auch noch in hess. der ratt. 
chratto, mhd. kratte, schwäb. kręto zu fassen. So fugt sich nun auch got. atta, mhd. Etzel (= urgerm. *att- aus *aðn-) gegen nhd. dial. ätte, däddi ${ }^{1}$ ) und wie die formen alle lauten, aus ${ }^{*} a b \delta$-, wie fries. aththa (Rustringen) vater Beitr. XI, 209 direct bezeugt. $\left.{ }^{2}\right)$ Für nhd. pocke hat ein germ. * pozo :*poho zu gelten, die assimilationserscheinung vertreten ags. pocc, nhd. pocke; ags. poca ist daraus wie ags. cnapa, haca s. 0. s. $532 \mathrm{f}$. zu erklären, während ags. pohha (vgl. Beitr. IX, $226 \mathrm{f}$.), bair. pfoche auf westgerm. geminiertes *poho- zurlickweisen. So nehme ich auch ags. moppe, mhd. motte, matte als in westgerm. periode gedehnt mit inl. $p$, während anord. motti, wenn nicht lehnwort, urgerm. die assimilation mitgemacht hätte. - Diese details durften gentigen, die belege obiger sammlung in meinem sinne zu erklären. Die mittel, die ich dazu in anspruch nehme, sind die beiden: 1. wechsel der stammhaften consonanz auf grund von Verners gesetz, 2. kurzung langer consonanz vor folgendem consonanten. Wenn die angesetzten kreuzungen scheinbar bunt sich ausnehmen, so beruhen sie doch schliesslich fast alle auf dem ubertritt der einen flexionsweise in die andere, im selben wort ubliche, oder auf dem umspringen der ableitung, das in der einen dialektgruppe nach der, in der andern nach einer andern seite erfolgen konnte, z. b. ahd. trocchan ist nur erklärlich, wenn neben der lautgesetzlichen form westgerm. *drozan- ein *drokk- bestand (s. o. s. 532 f.), d. h. bei der reproduction des sprechenden konnte, wenn *drozan im sinne lag, *drokk ins bewusstsein treten, und so die contamination *drokkan entstehen, oder bei der ab-

1) Der umlaut ist jungen datums, aber gerade bei verwantschaftsbezeichnungen häufig, vgl. schwüb. alem. bẹ's base, gotte, dotte pate neben gote, dotte patin, alem. schwäb. briodr = hruder etc., danach möglicherweise aus dem plural stammend.

2) Es scheint nicht beachtet zu sein, dass wie hier im fries. der geminierte reibelaut belegt, so auch der einfache laut noch aus den afr. rechtsquellen nachzuweisen ist. Gerade der name des Hunnenkönigs ist uns in den Rüstringer gesetzen in dem sogenannten vorwort überliefert (vgl. Richthofen 8. 131 ff.), 8. 133 a, 2. 39 thi kining Ethela, thi Huncna kining und mit diesem namen ist zweifellos identisch nom. ethla (Rüstringen) Richthofen s. 73, z. 33 (weitere formen im wörterbuch s. 721) in der bedeutung urgrossvater (grossvater), vgl. Jac. Grimm, Kl. schr. $\mathrm{V}, 302$. VII, $71 \mathrm{f}$. 
sicht auf *drokk die weiterbildung von *drozan massgebend werden; das gemeinsame ist eben die bedeutungsidentităt. Es ist sehr wol denkbar, dass bei einer doppelformigkeit *drozan $:{ }^{*} d r o k k$, auch ein analogisches ${ }^{*} d r o z$ entstunde. Ich glaube dieser umgekehrte vorgang muss fur folgende fälle in anspruch genommen werden.

Es ist die herrschende annabme, das nhd. adj. fett sei ein ndd. durch Luther eingefuhrtes lehnwort der schriftsprache. Bei der allgemeinen verbreitung desselben in obd. mundarten ist mir dies sehr unwahrecheinlich und ich stimme Winteler s. 65 bei, der darin ein echt altmundartliches wort sieht, dem das von ihm beigebrachte blutt bloss, ebenso im schwăb. u. a. blutt bloss, siegerl. blott kummerlich entwickelt, vgl. blöde, ganz analog ist. Ich kenne noch ein adjectivum mit entsprechender 'unregelmässiger' stammform gleichfalls weitverbreitet: šep $\left.{ }^{1}\right)$ schief, $p$ ist hier gegen die lautverschiebung, wie tt in fett, blutt. Fur alle drei worte sind aus andern oder denselben dialekten verwante, annähernd gleichbedeutende adj. nachzuweisen: fett: alem. feis - mhd. veiz, anord. feitr; blutt: $b l \bar{o} s=$ anord. blautr; šep : anord. skeifr schief. Wir sind in der lage etymologische entsprechungen in den andern idg. sprachen wenigstens fur $\mathrm{zwei}$ dieser worte sicher nachweisen zu kōnnen. fett ist längst zu abulg. pitati năhren, futtern ge-

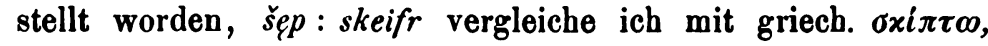
$\sigma x i \mu \pi \tau \omega$ (wurz. skeip) krumm, schief machen, hierzu lat. Scīpio $=$ der schiefe. $\left.{ }^{2}\right)$ Die idg. tenuis der grundwörter hat stets anstoss erregt. Schon die bedeutung von anord. feitr, mhd. veiz fett $=$ gefuttert, genăhrt (abulg. pitali năbren, futtern) macht es wabrscheinlich, dass das adj. aus dem alten -noparticip hervorgegangen ist, als dessen grundform * faiðno- zu vermuten ist (vocalstufe, wie der inlautende reibelaut entsprechen ahd. tougan occultus Beitr. IX, 181, vgl. auch ags.

1) Winteler a. a. o. nennt eine bildung bluts, ebenso ist im. schwäb. nicht šẹp sondern šeps allein üblich, am Niederrhein schäbb, siegerl. schabb (Heinzerling s. 73); ausl. -s ist offenbar die adjectivische neutralendung, die sich strichweise verallgemeinert hat, möglicherweise auch, wie in blutsnachtig bei Winteler, das adverbial -s.

2) $\mathrm{Zu}$ bloss stellt Kluge im Etym. wb. 8.32 griech. $\varphi \lambda v \delta \alpha \dot{\alpha} \omega$ zerfliessen, dial. blutt wäre nur damit vereinbar, wenn $\delta=d h$. 
hréac, anord. hraukr aus *hraugnó 0. 8. 515), dieses entwickelt sich regelrecht nach Kluges gesetz durch *faitta zu anord. feitr = mhd. veiz mittelst kürzung der geminata nach diphthong. Bei einer ei-wurzel wåre die regelmăssige participialform

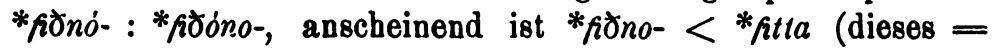
ndd. fett, mndl. vet, vettes?) durch eine nicht ersichtliche analogische einwirkung zu *faiðnó- $>$ *faitta geworden (möglicherweise bestand ein bcdeutungsgleiches, wurzelhaftes adj. mit vocaletufe -ai-, vgl. unten blautr, skeifr); einem *iঠóno- sollte westgerm. *fëठan-, mit $n$-dehnung ahd. *ëttan correspondieren. Damit ist das heutige fett nur zu vereinigen, wenn die kurzere stammform vorahd. *feit unter einfluss ron *fëtlan zu fëtt gewandelt, oder * fëttan nach feit, feiz, zu fëtt gekturzt worden ist.1) So muss auch bloss aus einem *blauðnó- (fur *bluðno?) $>$ *blautlo $=$ anord. blautr, ags. bleat, ahd. mhd. blöz entstanden gedacht werden, vgl. anord. blaupr, ahd. blodi Kluge s. 184, während blutt auf *bluðéno- > westgerm. *bluðin, ahd. mit $n$-gemination *bluttin zurlickzufuhren ist, dessen ableitung nach massgabe von blōz drangegeben worden zu sein scheint. Einfacher liegt die sache für anord. skeifi : dial. šep schief. skeifr ist unmittelbar an die zu grunde liegende wurz. skeip (s. o.) anzuschliessen, und westgerm. *skëban (aus urgerm. *skitóno-) fuhrt mittelst n-gemination zu ahd. *skëbban, *skëppan; md. schief, ndl. scheef zeigen, dass das wurzeladjectiv auch auf dem continent existiert hat, nach dessen muster schëbb, schëpp in derselben weise wie fëtt, blutt entstanden ist.

Ich glaube, diese annahme einer ableitungsverallgemeinerung noch durch weitere belege stutzen zu köunen. Griech.

1) Es ist noch zu beachten, dass daneben auch das particip des denominativen von * failta- gebildeten *failjan bestand: ags. faeted, faett, ahd. feizit, mhd. veizet, veizt. Eine synkopierte form *faild- muss bereits westgerm. existiert haben und mochte gleichfalls zur entwicklung von * fétt aus *féttan beitragen. Allerdings könnte *fëtt auch aus eben diesem *faitd anstandslos hergeleitet werden; die auslautende consonantenverbindung ist wie in den bekanntlich unverschoben gebliebenen mhd. satte, part. gesat gesetzt, zieht man noch die mehrfach eingetretene reduction des diphthongs vor doppelconsonanz bei (vgl. elf, heftig u. a.), so würde fëtt genan der voraussetzung entsprechen. Für blutt ist eine solche entwicklung ausgeschlossen. 
$\pi v \vartheta \mu \dot{\gamma}^{\prime} \nu$ steht aind. budhná, lat. fundus (ftr *fudnus, Ks. z8.

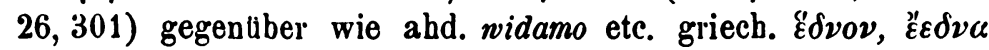
(hom.) für * ${ }^{*} \mathcal{E}_{\varepsilon} \delta o v$ (Bezz. Beitr. V, 240), worauf diese gewiss bereits idg. differenz der ableitungen gegrundet ist, bleibt unklar. Aind. budhná entsprăche einem german. *bott. Wie ahd. bodam ausweist muss ein german. *búbmo- existiert haben, dessen -m-suffix mit *bott- combiniert engl. bottom, ags. botm ( $t$ aus $t t$ vor consonant) ergeben hat, und so wird auch auf rein lautlichem wege anord. botn aus *botmr entstanden sein (ahd. bodam, ags. bopem Literaturbl. 1887, 3, 114; byðme Sievers, Ags. gr. ${ }^{2} \S 196,2$ anm. 2, as. bodm- setzen die stammbetonte form fort). In griech. E̊dvov scheint $d h$ zu $d$ geworden zu sein nach dem aspirationsgesetz ( lit. vediu fuhren, beimfuibren u. a.), einer entsprechenden m-ableitung entspricht genau ags. weopum, ahd. widamo, mhd. wideme, widen; dagegen ist auf grund des vorangehenden falles

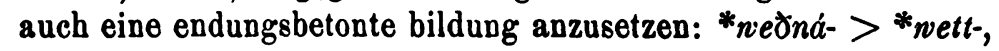
deren consonanz mit der nebenform combiniert zu $t$ in ags. weotuma, afries. wetma, witma fuhren konnte (got. *witma), vgl. noch wittemo der lex. Burgund. -

Diese letzten beiden falle haben zur gelm. assimilation zurluckgefuhrt; ich hebe nochmals hervor, diese beruht a uf ganz andern bedingungen als die westgerman. $n$-dehnung und an einen zusammenhang zwischen beiden erscheinungen darf nicht gedacht werden. Die kreuzungen, dio wir verfolgt haben, sind eingetreten, nachdem die wirkungen des assimilationsgesetzes längst ábgeschlossen waren, wenn die $n$-dehnung nomina betroffen hat, die urgerm. assimilation resp. gemination erfahren hatten, so rangieren dieselben mit denjenigen, bei denen dies nicht der fall war, in derselben linie. Eine phonetisch-physiologische begrlindung der urgerm. assimilation eines folgenden nasals an den vorangehenden geräuschlaut vermag ich nicht zu geben, der vorgang ist mir unklar.

Dagegen liegt es nahe, zu ermitteln, wie der process der westgerm. n-dehnung sich abgespielt haben könnte. Da dieselbe genau in ihren wirkungen mit der dehnung vor $i, n$, $r, l$ zusammentrifft, muss die erklärung auch für diese laute gelten. Ich glaube folgende theorie der westgermanischen consonantendehnung aufstellen zu mussen. Dio 
lauterscheinung, dass vor $i, w, r, l, n, m$ (8. 541) vorangehende consonanz gedehnt (resp. verdoppelt) wird, ist ein vorgang gewesen, der, wie wir heute noch beobachten, mit der function des einzelnen wortes zusammenhing. Beim substantiv und adjectiv sind es immer nur einzelne casus, welche die lautliche verånderung erleiden, beim verbum gleichfalls nur einzelne formen. Das lautgesetz, wenn ich es so nennen will, betrifft nicht den consonantenstand als solchen. Daher sehen wir, dass die etymologische inl. consonanz des wortes nur auf dem wege der association untergehen konnte. Got. hvaiteis weizen musste zu mhd. weize fuhren, wenn das wort heute in verschiedenen mundarten inl. -ts- zeigt, konnte dies nur durch die präponderanz geminierter formen (vgl. dat. *hvaitia $>$ hvaitja $>$ *hvaittja) bewirkt werden und zwar erst allmăhlich, ursprttnglich bestand in einer und derselben individualsprache ein hvaitī : (*hvaití- $<)$ *hvaitti-. Dieser wechsel zieht sich durch die ganze einschlägige kategorie hindurch.1) Man ist sogar geneigt gewesen an der geminierenden wirkung von $w, r, l \mathrm{zu}$ zweifeln, weil 'die beweise fur den mangel einer wirkung, welcher der des $j$ entsprăche, zahlreich' sind, Beitr. VII, 106 anm., ebenda findet Paul die doppelschreibungen blō ddre neben blä dre, nae-ddre neben noe-dre nicht klar; sie ruhren eben daher, dass in den flexionsformen in denen $d$ und $r$ zusammentraten $d d$ entwickelt wurde, während nach massgabe nicht-synkopierender formen der einfache laut in der oder jener sprache sich verallgemeinert hat, nachdem das gesetz erloschen war. Vgl. Sievers, Ags. gr. ${ }^{2} \& 228$ nebst anm. 229. Es ist mir nicht ein einziger fall bekannt, wo dehnung eingetreten

1) Diese tatsache ist bis jetzt leider verkannt worden, trot\% Paul, Beitr. VII, $112 \mathrm{ff}$, und es hat dieser umstand namentlich in dialektdarstellungen $\mathrm{zu}$ leidigen ungeheuerlichkeiten gefubrt und ein sachgemässes verständnis der lauterscheinungen verhindert, ich nenne ein beispiel für vielc. In der vortrefflichen darstellung Scheiners (Die Mediascher mundart, oben 8.113 ff.) steht 8.142 'in der gemination ist $p$ in alten dialektwörtern unverschoben geblieben'. S. 143 werden dafür 'apl (apful), tsop (zopf)' u. a. beigebracht, aber als anm. 2 wird angehängt: 'verschoben ist $p p$ in lehnwort k'afr (kupfar)'. Wie ags. copor neben ahd. chupfar, auf einen wechsel der stammformen *cupur-, *cupr- deuten, so ist auch im siebenb. $f$ regelrechter vertreter des einfachen $p$. 
wäre, wenn $i, w, r, l, n$ in såmtlichen wortformen unmittelbar auf den betr. consonanten folgte, vgl. Paul, Beitr. VII, 112 ff. Ich erinnere an die jan-verba, 2. 3. sg. präs., präteritum, particip. praeteriti hatten im wortkörper kein $i$, vgl. Sievers, Beitr. V, $126 \mathrm{f}$, bei den nominalen jo-stämmen war dat. pl. (vgl. Beitr. VI, 221. VII, 113. 161 f.) und der nom. sg.1) von der dehnung ausgeschlossen, in der schwachen -jon-declination musste das $i$ der ableitung vor dem genet. dat. sg. -en, -in fallen, und bei den femininen lautete bekanntlich der nom. sg. auf $-\bar{i}$, vgl. Sievers, Beitr. V, 136 ff., Paul, Beitr. VI, 164. VII, 113, bei $w$ (vgl. z. b. ags. nacot $=$ ahd. nahhot, mfr. nachet Beitr. I, 24 : got. naqaps = ahd. nacchot nackt), $r$ (vgl. ags. accer $=$ schweiz. achar : abd. acchar, nom. *akar, gen. *akres u. s. w.), $l$ (vgl. ahd. leffil : ahd. lepfil löffel u. a.), $n$ (vgl. abd. rabo : rappo rabe ete.) ist die erscheinung des wechsels za bekannt. Danach muss der gesetzmãssige verlauf des vorgangs gerade in diesem wechsel gesucht werden, und dieser wechsel ist der anlass einer umbildung. Das typische der zustände druckt sich in folgenden formeln aus; wobei ich

1) Sievers, Ags. gr. $\$ 130$ anm. setzt für ags. secz einen nominativ westgerm. *sazzjoz (ebenso *kunnjo = ags. cyn) aus germ. *sazjoz voraus, wie er das Beitr. V, $125 \mathrm{ff}$. näher begrtindet hat, vgl. anch Paul, Beitr. VI, 162. Die entwicklnng ist nur begreiflich, wenn man mit Sievers, Ags. gr. a. a. 0. annimmt, dass $j$ bereits vor der 'apokopo' des nominativ. -0- geschwunden ist, aus dem ags. selbst wisste ich nichts zweifelloses vorzubringen, dagegen sind aus dem as. und ahd. die belege fur bewahrung des $j$ so zahlreich nnd bekannt, dass ich mit hinweis anf Paul, Beitr. VII, 106. VI, 149 dieser annahme von Sievers mich nicht anschliessen kanp. Ich glaube vielmehr, dass die einzig moglichen formen igs. *seze, *hryze, as. *hrugi etc. (aus westgerm. "saziz, *hruziz) gewesen sein kơnnen. Fur den umlant des nom. milsste unter allen umstïnden analogiebildung nach den obliquen casus angenommen werden. Im ags. (vereinzelt im as.) ist der nominativ m. e. nach massgabe dieser casus in die flexion der $i$-stämme tibergefuhrt worden, nach dem muster der ubereinstimmung zwischen wyrmes : hryczes entstand der nom. hrycz wie wyrm n. s. w., in gleicher weise bei den neutris; im as. sind mit ausnahme von bed, eämtliche nomina in der jo-flexion verblieben aber die doppelconsonanz der obliquen casus ist auch in die nominativform gedrungen, *hrugi $>$ hruggi nach* *hruggies, ebenso middi etc. In got. harjis, nipjis etc. halte ich demnach nur $j$ für unursprtinglich, $j o$ ist durch $i$ vertreten. 
mit den auslautenden vocalen uberhaupt nur vocalisch anlautende endung bezeichne:

tala : talia, naka : nakua, aka : akra, lapa : lapla, raba : rabna, d. h. mit andern worten, der silbentrennung $t a-l a$, steht die von tal-ia gegentiber, ebenso na-ka: nak-ua, $a-k a$ : ak-ra, la-pa : lap-la, raba : rab-na; wenn nun tal-ia : tallia, nakua : nakkua, ak-ra : akkra, lap-la : lappla, rab-na: rabbna wird, so ist das nichts anderes als was man mit verschiebung der silbengrenze bezeichnet bat. ${ }^{1)}$ Dass der ausgleich der bestehenden differenz der silbenteilung (die contamination) nach massgabe des typus ta-la (tal-ina $>$ tal-lia $a$ ) erfolgt ist, beweist der bestand unserer ältesten uberlieferung. 1. Der typus $t a-l a$ ist nicht zu grunde gegangen sondern intakt geblieben, $\nabla g l$. abd. zelis, heuis etc. 2. Der typus tal-įa ist umgestaltet worden, vgl. ahd. zellu, heffu etc. Die lautvorstellung ta-la war also die mächtigere. Wenn ich nach meinem sprachgefuhl urteilen darf, so wurde ich das product nicht als eigentliche geminate betrachten, sondern als lange consonanz, vielleicht mit urspranglichem untergeordnetem, eigenem silbenaccent, der aber in der lautentwicklung verloren gegangen ist, wie wir dasselbe häufig beobachten

1) Dass die silbentrennung wirklich in dieser weise bestanden hat, wird ganz unzweideutig durch die rhythmische verwendung dieser lautcomplexe in der alliterationstechnik berwiesen. Nach Sievers' untersuchungen fungiert eine lautgruppe aka stets als a-ka (mit kurzem erstem sonsnten), während in akra eine erste geschlossene (rhythmisch lange) silbe ak-gegolten hat. Die ausdrilicke silbentrennung, verschiebung der silbengrenze sind nur von mir gewăhlt, weil sie der allgemein phonetischen terminologie angebören. Der sprechende ist sich einer trennung ron silben so wenig bewusst als er die abstraction dessen, was wir unter silbe $2 u$ versteheu plegen, vollzöge. Im grunde handelt es sich vielmehr darum, dass im bewegungsgefühl für eine bestimmte lantgruppe (wort) eine differenz zu tage tritt, die ausgeglichen worden ist. Der absatz des vocals in offener silbe ist, nach unsern verhältnissen zu urteilen, doch kanm ein anderer als der leise oder leise gehanchte gewesen $(a-k a)$, vielleicht bei schwach geschnittenem accent; während wir für die geschlossene silbe festen absatz voraussetzen mulissen. In dieser differenz der kehlkopfarticulation bei der exspirationstätigkeit sehe ich die physiologische grundlage für die sogenannte 'verschiebung der silbengrenze'. 
können, gerade in fällen wo es sich gleichfalls um eine verschiebung der silbengrenze handelt.1)

Dieser theoretische excurs war aus zwei grluden an dieser stelle notwendig, einmal weil auf diese weise erklärt wird, warum $-n$ in einer grossen zabl von fällen keine dehnung hinterlasseu hat und dann, damit zusammenhängend, warum wir nur ganz vereinzelt dehnung vor $-m^{2}$ ) constatieren können.

1) Ich nehme als beispiel: schwäb. $r$ falt $=$ er fält, wird in der mebrzahl der fälle mit langem $l$ gesprochen, das ältere und von mir auch noch mehrfach beobachtete ist aber gewiss rfa-lt wobei $l$ den silbenaccent des syncopierten vocals übernommen hat. Wenn wir diesen untergeordneten silbenaccent voraussetzen, gibt er auch die moglichkeit an die hand, die in gewissen strichen eingetretene eigentliche geminate mit exspirationspause (vgl. z. b. Stickelberger, Mundart von Schaff hausen 8. $12 \mathrm{ff}$.) zu erklüren. In pausastellung ist bei sonoren vielfach unter wirkung dieses secundären accentes svarabhakti o eingetreten, die bereits von Sievers als auf verschiebung der silbengrenze beruhend erkannt ist. - Sievers, Beitr. V, $161 \mathrm{f}$. hat die gemination auf circumflectierende betonang zurtickgeführt, dieselbe ist für kurzen vocal an sich doch unwahrscheinlich, bei langem vocal oder diphthong nicht ansreichend; die beschreibung des vorgangs der dehnnng des consonanten trifft aber so sehr mit dem resultat der 'verschiebung der silbengrenze' zusammen, dass ich seine worte mir herzusetzen erlaube: 'der zweite teil des accents (d. h. in meinem sinne der secundär-accent) fällt in den eingang des anf den rocal folgenden consonanten und verlängert diesen selbst; der augang des consonanten aber wird nach wie vor zur folgenden silbe gezogen und so entsteht der eindruck der geminata'. Vgl. noch den abschnitt 'silbentrennung' in Sievere Phonetik.

2) Einige wenige fälle sind mir bekannt geworden, die wol nur unter annahme einer dehnung vor $-m$ erklïrt werden können. Im siebenbürgischen wie am Niederrhein (auch Remscheid, Holthausen, Beitr. X, 421, Soest, Soester ma. $\$ 168,3$, an beiden stellen hat H. -m- als 'stimmlosen' consonanten geñommèn) list intersonantisches altes -s-stets stimmhaft (z); in beiden sprachgebieten bildet unerklärbare ausnahme das wort für besen, z. b. siebenb. biosm (nicht *biozm) oben 8. 141 (Mediascher mundart $\$ 93 \mathrm{anm} .1$ ), ebenso mnl. bessem neben bẽzem. Wie in den ebenda namhaft gemachten gisl, hasl (und in denselben worten gleichfalls am Niederrhein) $s$ nur aus -ss- erklärbar ist (als dehnung vor -l-) so muss ein wechsel westgerm. *bësum : *besm zu -ss- gefuhrt haben (vgl. Franck, Mnl. gram. 8. 14), dessen reflex in sieb. biosm etc. vorliegt. Bei ags. máðum ist gleichfalls aus alter zeit der wechsel $m \dot{a} \delta m: m a ̈ \delta u m ~ b e-$ zeugt, vielleicht beruht auch hier mádrum auf gemination vor $-m$, doch vgl. K8. z8. 26, 99. Sicher scheinen mir auch die mnl. nndl. ascm, 
Im westgerman. ist nur in den verbindungen $(r, l+$ consonant resp.) consonant $+r, l$ svarabhaktivocal eingetreten ( $\nabla g l$. Beitr. VIII, 328 anm.), fur $n, m$ haben wir wenigstens nach kurzer silbe keine directen zeugnisse. Ich kenne nur ags. begn, as. thegn Hel. (neben thegan), ags. swefn, ags. hraefn, ags. stefn, rezn, waezn vgl. Sievers, Beitr. V, 80; ebenso trosm rauch, faeठm umarmung, botm boden (daruber 8. 0. s. 537) vgl. Sievers, Beitr. $\mathrm{V}, 80$; waestm wachstum, doch findet sich nach Sievers auch waestem u. a., uber die langsilbigen vgl. Sievers, Beitr. V, 72, und gewőhnlich mátum, ebenso ist neben ${ }^{*} \bar{a} p m$ ein $* \bar{a} p u m$, vorauszusetzen und das oben s. 541 anm. 2 angefuhrte vereinzelte *besum neben *besm, *fapum neben * fapm beizufugen, vgl. Sievers, Ags. gram. ${ }^{2} \S \S 141.142$. Beitr. X, 481 haben sich als rhythmisch erforderlich bósm, mäঠm, beácn, tácn, waepn, wolcn ergeben, dagegen einmal (mit gemination) mádరum (s. o.). Unter diesen langsilbigen bildet bósm die einzige ausnahme, fur welche ich noch keinen beleg der dehnung beibringen kann, alle andern hier genannten nomina mit langer erster silbe haben solche erfahren ( s. 522, schweiz. wäffo s. 526, schweiz. wulkchə s. 524), und es muss daraus geschlossen werden, dass nach kurzer silbe westgerm. schliessendes $-m, n$ meist kein vocaltimbre entwickelten. Demnach galt im gesammten formsystem nur die silbe faedm, ठegn, hraefn, stefn ${ }^{1}$ ), rezn ${ }^{2}$ ) etc., die silbentrennung war durchweg dieselbe (die stanmsilbe geschlossen), eine verschiebung der silbengrenze konnte also uberhaupt nicht eintreten, weil der wechsel zur

aessem neben adem, ebenso ahd. atum $(t=t t)$ atem, asemen neben ademen und vasemen, vessemen neben vademen mit arinen umspannen Franck, Mnl. gr. § 95 anm. 2, Grimm Gr. (neudr.) 8. 459 auf gemination von $b$ vor $m$ zurtickgefuhrt werden zu misssen, $\nabla g l$. nl. $k$ klis $=$ abd. chletta Kluge s. 160.

1) In diesen fallen, in denen kein wechsel zwischen - fn- und $-f+$ vocal + n- statt hatte, ist die bekannte reciproke assimilation von - $f n$ $>m n$, mm erfolgt: ags. em, emn, sus efn eben, hrem, hraem (hremmes) aus hrafn rabe, stefn, stemn (got. stibna) u. \&. Sievers, Ags. gr. $\$ 188,1$, doch vgl. \$193 anm.; ebenso wol auch $p n>m n$ in waémn aus vaépn nebst ableitungen.

9) Vgl. die tánc, renz, Jenz, frenz Sievers, Ags. gr.` § 185; waen, rén, Jén $\$ 214,3$. 
zeit der dehnugserscheinung nicht herrschte, und so liegt hierin der einleuchtendste grund, warum in diesen wörtern keine doppelschreibung begegnet. Dass in späteren perioden sich auch nach den kurzsilbigen svarabhakti entwickelte, ist dafür ganzlich irrelevant, doch scheint es mir glaublich, dass auch nach kurzem vocal bereits westgermanisch unter entsprechendem satzrhythmus die svarabhakti eintrat, wie ich dafür das aus niederrb. siebenb. biasm erschlossene westgerm. *besum ebenso * fabum nach mndl. vasem s. $541 \mathrm{f}$. anm. als letzte zeugen geltend machen möchte. Für die svarabhaktientwicklung bei $r, l$ nach kurzer silbe muss gleichfalls der satzzusammenhang (verteilung der nebentöne) massgebend gewesen sein und es bestanden westgerm. doppelformen *apul aber *fogl u. a., im letzteren fall war widerum die dehnung ausgeschlossen; dieselbe anschauung gilt fur consonanz $+\underset{\sim}{\sim}$, dehnung ist in der regel nur eingetreten, wo alte suffixabstufung zu grunde liegt, vgl. Beitr. VII, 163. -

Der ursprung der german. assimilation ist nach seinen bedingungen von Kluge Beitr. IX, $168 \mathrm{ff}$. in der existenz teils eines betonten $n \delta$-suffixes, teils der schwachsten stammform der schwachen declination (masculinen wie femininen vgl. s. 177) nachgewiesen worden, vgl. Osthoff, Beitr. VIII, 299 f. anm. Dass beztiglich der morphologie bei dem no-suffixe gleichfalls abstufung -ono, -eno, -no statt hatte, ist bekannt genug und ist uns in den materialien mehrfach begegnet; es darf nur fur die -no-stufo assimilation vorausgesetzt werden. In der schwachen flexion war urgerm. zweifellos noch der genet. plural. mit dem tiefstufigen ${ }^{*} n \delta, *_{n \bar{e}}$ gebildet (got. auhsne, $a b n \bar{e}=$ run. auna? auf dem Bracteat von Sjaelland), ich sebe vou den sehr zweifelhaften gen. dat. und instrum. sg. ab, bin aber geneigt anzunebmen, dass auch im dat plural. (vgl. got. abnam, valnam) wenn auch nicht durchweg, so doch unter umständen die $n$-ableitung platz gegriffen hatte, wio ich auch auf grund des von Kögel, Beitr. VIII, 115 restaurierten got. auhsnuns $\left.{ }^{1}\right)$ 1. Cor. 9, 9, vgl. ebenda got. bröpruns, die existenz eines

1) Als sebr gewichtige instanzen fur diese form scheinen mir die anord. nom. yxn, $\theta x n$ sus *uhsniz, zu sprechen, von dem einzigen genetiv pluralis aus hätte die verallgemeinerung dieser schwächsten stammform kaum wahrscheinlichkeit, vgl. ags. axen (aus *oksenez).

Beiträge zur geechichte der deutschen sprache. $\mathbf{X I I}$. 
'schwăchsten' acc. pluralis betonen möchte. Es ist namentlich von Osthoff in seinen untersuchungen uber die germ. schwache declination gezeigt worden, dass wir uns dieselbe in einem zustande zu denken haben, der noch möglichst viel von dem charakter des idg. prototyps an sich trăgt und seitdem haben Noreen, Beitr. VII, 431 ff., Leffler, Tidskrift, N. F. IV, 285, Kluge K8. zs. 26, 92 ff. mit dem nachweis der fortdauer des wechsels zwischen wurzel- und endungsbetonung fur diese anschauung gewichtige argumente beigebracht. Mit diesem accentwechsel war 2 weifellos auch noch die alte vocalische abstufung des ablauts der wurzelsilbe lebendig und wir sind berechtigt, die verschiedenen vocalstufen, die wir historisch auf etymologisch identische aber meist nach der bedeutung differenzierte nomina verteilt sehen, in einem und demselben urgerm. paradigma zu vereinigen. Einzelnes ist bereits in den ebengenannten aufsătzen verwertet worden; vgl. ferner anord. floki : ahd. floccho, ags. flöh : schweiz. flock, anord. fraukr : ags. frocca, analog anord. flik : ahd. flëccho; mhd. knouf : ahd. chnopf, ahd. kloz, engl. cleat : mhd. klotz, engl. clot dazu noch hess. klüte, klaute; $0: e$ in ahd. nacch : ags. hnecca, ags. haef : schweiz. hëpf, ags. gnaet : nhd. gnitze, ahd. starz : stërz, ags. steort, ahd. chralto : chrëzzo; $e: r$ in ahd. krëta : krota kröte; ou : $\bar{u}$ in ahd. houf : hüfo baufe; ags. hreac, anord. hraukr : hruga; $\bar{u}: u$ in anord. knütr : ahd. chnodo; $\delta: \bar{e}$ in anord. $k r \delta k r$ : anord. krákr, ahd. chrāgo; $\delta: \partial$ in ags. hōd : ags. haett, anord. hottr, got. lofa : ahd. laffa; $\bar{e}:$ a in chrāpfo : schwäb. krapfo, schwab. wöfo : wappen; $\bar{\delta}: \bar{e}: \partial$ in ags. $h \delta c:$ ahd. hago : ags. haca. Im gtunstigsten fall vermögen wir also noch dreistufigkeit zu belegen; sie ist als das allgemeine vorauszusetzen, die dreifache suffixabstufung (Paul, Beitr. VI, 114) in der consonantischen declination scheint ihr ganz parallel zu laufen, ohne dass damit ausgemacht wăre wie sich die einzelnen stufen verteilen (hé zon-: hozén : hazn-?).1)

1) Vgl. dazu noch ahd. scincho schenkel, mhd. schinke schenkel, schinken : ags. sceonc, sceonca beinröhre : afries. skunka schenkel, beinrơhre, diese letztere stufe auch z. b. in schwäb. sõøko ( $\tilde{o}$ aus $u$ ) schinken, die e-stufe bewahrt das afries. in berskinsze = nudipes Richthofen, Wörterbuch 8. 627. 1163, vgl. Grimm, Gram. Is 407. 
In gemeingerm. sprachperiode ist bereits der zustand eingetreten, wie er in den eiuzelnen zweigen vorliegt, d. h. von den abgestuften flexionsformen ist in der einen mundart diese, in der andern jene zu alleiniger geltung gekommen, wo doppelformen bestehen blieben, hat sich damit differenzierung der bedeutung verkn(upft. 1) Dass wir aber in westgerm. zeit fur die casusendungen die formen der alten abstufung, wenn nicht isoliert so doch jedenfalls ohne irgendwelche functionelle bedeutung ansetzen durfen, scheint keinem bedenken zu unterliegen. Got. auhsnē (auhsnuns s. o.) entspricht (von dem aus dem nominativ stammenden umlaut abgesehen) an. yxna, øxna, ags. oxna (vgl. auch Oxnaford in der Chronik, andd. Osnabrugga Grimm, Gram. I, 551 (neudr.), Arnahurst Heinzel, Nfr. geschspr. s. 28), der genet. pl. oxena ist erst jung bezeugt, mit den got. abnam, namnam, vatnam scheinen, was die suffixstufe betrifft, auf derselben linie zu stehen, anord. yxnom, $x-$ nom, ag8. oxnum in einer urkunde a. 858, bei Kemble cod. dipl. II, 64 f. (no. 281) = Sweet old. engl. Texts s. 437 f. (vgl.

1) Ich wage es daran eine allgemeine erwägung zu schliessen. Von unserem heutigen sprachgefuhl aus sträubt man sich in der regel für begrifflich identisches formal verschiedene lautformen anzuerkennen. Der sachverhalt ist nur nicht derart; ein wechsel der form hängt nicht an dem begrifflich identischen, sondern, so viel wir erkennen, an dem functionellon : der gen. sg. eines substant. hat in der sprechtätigkeit eine andere function als der gen. plural, so sehr beide auch die gemeinsam identische vorstellung der allgemeinbedeutung verbindet. Spuren dieses verhältnisses lassen sich heute noch reichlich in der gesprochenen sprache, besonders treffend an den sogenannten en- und prokliticis beobachten, deren form bekanntlich erheblich verschieden ist, je nachdem sie emphatisch oder nicht verwendet werden. Doch darf man behaupten, dass dieses sprachgefuhl das nur functionell verschiedene, lautlich zu variieren für uns nicht mehr existiert. Diese umbildung des sprachgefühls muss als historisches ereignis in die sprachgeschichte eingefugt werden. Während in der declination dieselbe als vollzogen längst hinter uns liegt, geht der process in der verbalflexion seit jahrhunderten langsam demselben ziel entgegen. Es liegt nahe die oben s. 537 ff. entwickelto theorie tiber die bedingungen der westgerm. consonantendehnung mit dieser erwägung zu verkntipfen, als letzter psychologischer anlass resp. anstoss zum ausgleich der silbenteilung, wäre die uniformierung des lautk8rpers auf grund der bedeutangsidentität zu betrachten, vielleicht ein letzter ausläufer dieses die nominalflexion von grund aus umgestaltenden vorgangs. 
Kluge, Beitr. VIII, 533 zu Beów. 1027), s. 0. s. 527 f., Beitr. XII, 94. Hierher gehören ferner ags. worðigna, dat. worðignum Kluge a. a. 0., geuet. pl. ags. cvenna (= anord. kvenna), ausserdem ags. cūna, cȳna der kuhe (cȳna wie anord. yxna), vgl. dat. beonum, fānum bei Kluge a. a. o. Diese isolierten reste beweisen die existenz der schwåchsten suffixform $-n$ - in westgerm. periode, und zwar ist mir durchaus wahrscheinlich, dass anord. yxnom mit ags. oxnum (gegen oxum) (einer- und got. aúhsnuns (s. 0.) andererseits) nicht neubildungen nach den genet. pl. sondern die regelrechten dative plural. $\left(=-n m^{1}\right)$ ) darstellen, vgl. afries. āchnum, āchnon (Rustr. 29, 27) gegen ags. eázum. Die verbreitung dieser endungsform ${ }^{2}$ ) in den westgerm. einzelzweigen lässt sich nicht feststellen. Wenn wir auf der einen seite zeugen des $n$-suffixes, auf der andern die wirkungen desselben in zahlreichen fallen nachgewiesen haben, ist der schluss berechtigt, zum mindesten fur die nomina mit gedehnter consonanz vielleicht nicht bloss im gen. sondern auch im dat. plural. dasselbe vorauszusetzen. Auf grund von ags. oxna, anord. yxna, got. auhsne etc. vermute ich einen westgerm. gen. *balknō (dat. *balknum) > *balkkno, vgl. schweiz. balkcho

1) Mit der annabme, dass $n$ vor nasal nicht - $u$ - entwickelt habe, sondern dass assimilation (acc. sg.) eingetreten sei, streitet diese ansetzung. Ich halte die assimilation nur für möglich, wenn die betr. silbe im satzzusammenhang vollig nachdruckslos geworden war, so lange der silbenaccent gewahrt blieb, musste n zu un, nu sich entwickeln. Zu vergleichen ist die geschichte secundärer diphthonge wie in ahd. sẽu, sẽo, got. saiv-; einsilbiges seo, sio kann nur in bestimmtem satzrhythmus, in dem $u$ den nebenaccent verlor, entstanden sein; seu, seo und seo, sio haben als satzdoppeltormen zu gelten wie nm $>$ nun, oder $>n . \quad$ Vgl. noch Sievers, Beitr. V, 158 anm.; 160.

2) Nehmen wir such an, dass der gen. pl. in westgertm. oder vorwestgerm. sprachperiode zweisilbige endung gehabt bätte, so musste nach den westgerm. Bynkopiernngsgesetzender mittelvocal unter bestimmten bedingungen synk opiert werden. Diese moglichkeit ist von Moller, Beitr. VII, 534 ff. ganz ausser acht gelassen worden, die ags. gen. pl. arna, lärna, sorzna, eázna sind wol kaum anders zu erklären. Ahd. -ono ist sicher neubildung, nur lässt sich nicht entscheiden, ob die angleichung an den nominativ die alte oder jtingere einsilbige ondung betroffen hat. Fuir die $n$-dehnung k $8 n n e n$ die resultate des synkopierungsgesetzes nicht beigezogen werden, weil, von allgemeinen grïnden abgesehen, ther die chronologie der erscheinungen nichts auszumachen ist. 
(vgl. balchə), und wie ags. cvenna, cūna, cy na wird ein westgerm. ${ }^{*}$ foznō < ${ }^{*}$ fozznō, vgl. ags. focze fluchsin (zu got. fauho), bestanden haben, dasselbe gilt fur die ubrigen feminine wie harpfe, seipfe, flù ahd. hëlpfa erinnere ich an die in der alliterationsdichtung so sehr beliebte verwendung des gen. pl. u. s. w. Es verdient noch hervorgehoben zu werden, dass die meisten schwach flectierenden nomina mit n-dehnung kurzsilbige stämme sind, gen. pl. *dropn $\delta>{ }^{*} d r o p p n \delta$ in ahd. tropfo, wie got. $a b a$ : $a b n \bar{e}$ (dieses durch siegerl. abbe etc. s. 0. 8. 515 vorausgesetzt), got. namna, namne, namnam, watnam : namô, watô. MARBURG. FRIEDRICH KAUFFMANN. 